\title{
Quantum Phase Estimation Algorithm with Gaussian Spin States
}

\author{
Luca Pezzè ${ }^{*}$ and Augusto Smerzi ${ }^{\dagger}$ \\ QSTAR, INO-CNR and LENS, Largo Enrico Fermi 2, Firenze 50125, Italy
}

(Received 10 October 2020; revised 21 April 2021; accepted 30 July 2021; published 1 October 2021)

\begin{abstract}
Quantum phase estimation (QPE) is one of the most important subroutines in quantum computing. In general applications, current QPE algorithms either suffer an exponential time overload or require a set of - notoriously quite fragile - GHZ states. These limitations have so far prevented the demonstration of QPE beyond proof of principles. Here we propose a new QPE algorithm based on a cascade of Gaussian spin states (GSSs) and a suitable adaptive measurement protocol. GSSs are renownedly resilient and have been created experimentally in a variety of platforms, from hundreds of ions up to millions of cold and ultracold neutral atoms. We show that our protocol achieves a QPE sensitivity overcoming previous schemes, including that obtained with GHZ states, scales linearly with time, and is robust against certain sources of noise and decoherence, such as detection noise, in particular. Our work paves the way toward an efficient implementation of the QPE, as well as applications of atomic squeezed states for quantum computation.
\end{abstract}

DOI: 10.1103/PRXQuantum.2.040301

\section{INTRODUCTION}

Quantum phase estimation (QPE) [1-3] is the building block of known quantum computing algorithms providing exponential speedup [4], including the computation of the eigenvalues of Hermitian operators [5], such as molecular spectra [6,7], number factoring [8-10], and quantum sampling [11]. All these applications require the calculation of an eigenvalue of a unitary matrix $U|u\rangle=e^{i \theta}|u\rangle$, where $|u\rangle$ is the corresponding eigenstate. This problem can be cast as the estimation of an unknown phase $\theta \in[-\pi, \pi)$, where the information about $\theta$ is encoded into one or more ancilla qubits via multiple applications of a controlled- $U$ gate [4]. The QPE problem plays a key role also in the alignment of spatial reference frames [12] and clock synchronisation [13], with further developments in atomic clocks [14], and clock networks [15].

According to the current paradigm [1,2], QPE algorithms are implemented iteratively, without requiring the inverse quantum Fourier transform [3,4,16,17]. Iterative QPE consists of multiple steps, each step being realized in two possible ways. (i) Using a single ancilla qubit $(|0\rangle+|1\rangle) / \sqrt{2}$ that interrogates $2^{k}$ times the controlled$U$ gate in temporal sequence [18-20]. In this way, the

\footnotetext{
*luca.pezze@ino.it

†augusto.smerzi@ino.it
}

Published by the American Physical Society under the terms of the Creative Commons Attribution 4.0 International license. Further distribution of this work must maintain attribution to the author(s) and the published article's title, journal citation, and DOI. ancilla qubit is transformed to $\left(|0\rangle+e^{i 2^{k}}|1\rangle\right) / \sqrt{2}$ and the phase information is extracted via a Hadamard gate $H$, followed by a projection in the computational basis. (ii) Using $2^{k}$ ancilla qubits in a Greenberger-Horne-Zeilinger (GHZ) state $\left(|0\rangle^{\otimes 2^{k}}+|1\rangle^{\otimes 2^{k}}\right) / \sqrt{2}$ that interrogate the controlled$U$ gate in parallel $[14,15,21-23]$. In this case, the GHZ state is transformed to $\left(|0\rangle^{\otimes 2^{k}}+e^{i 2^{k} \theta}|1\rangle^{\otimes 2^{k}}\right) / \sqrt{2}$ and the phase can be extracted by applying a collective Hadamard gate $H^{\otimes 2^{k}}$ followed by a parity measurement [21-23]. While the above output states are characterized by a periodicity $2 \pi / 2^{k}$ in $\theta$, an unambiguous estimate of $\theta \in$ $[-\pi, \pi)$ is obtained by taking a sequence of steps using $k=0, \ldots, K$ and eventually repeating the procedure an optimal and finite number of times $v$ at each step $[1,2]$. Using total resources $N_{T}=v \sum_{k=0}^{K} 2^{k}=v\left(2^{K+1}-1\right)$, it is possible to estimate an unknown $\theta$ with a sensitivity reaching the Heisenberg scaling $\Delta \theta \sim 1 / N_{T}[18,23,24]$.

These protocols have critical drawbacks. The sequential protocol (i) considers $2^{k}$ applications of the controlled$U$ gate that require exponentially large implementation times. This approach has been demonstrated experimentally [18] using multiple passes of a single photon through a phase shifter and it has been more recently applied to eigenvalue estimation [19] and magnetic field sensing [20]. The parallel protocol (ii) scales linearly with the implementation time but requires GHZ maximally entangled states containing an exponentially large, $2^{k}$, number of particles. These states are notoriously fragile, since the loss of even a single particle completely decoheres the state $[25,26]$ and irremediably breaks down the quantum algorithm. GHZ states are currently implemented with up to 20 particles [27,28]. Generally speaking, the current 
grand challenge in quantum technologies is to go beyond proof-of-principle demonstrations toward implementations overcoming "classical" bounds. In the contest of QPE, it is evident that present shortcomings call for a radically new approach that is both scalable with respect to temporal resources and exploits experimentally robust, easily accessible, quantum states.

Here we develop a new QPE algorithm that is implemented with Gaussian spin-squeezed states (GSSs) [29-31] and a suitable adaptive measurement protocol. The circuit diagram of the quantum algorithm is shown in Fig. 1 and is discussed in detail below. The iterative algorithm scales linearly with time and is broken into multiple steps, each implemented with a GSS. GSSs have a Gaussian particle statistical distribution and are way more robust to noise and decoherence than GHZ states. Indeed, GSSs have been experimentally demonstrated in a variety of platforms [30], with a few hundreds ions [32], several thousands degenerate gases $[33,34]$, up to millions of cold [35-38] atoms. Recent experiments have reported the generation of GSSs with long lifetimes (up to 1 s) [39] and a large (up to $10^{11}$ ) number of particles [40]. The shortcoming of GSSs - that has prevented so far their use in QPE - is that they provide high sensitivity only in a relatively small phase interval centred around the optimal phase value $\theta=0[30,31]$. Our algorithm overcomes this limitation by a classical adaptive phase feedback. It uses a cascade of GSSs to progressively drive the unknown phase to the optimal sensitivity points of a final highly populated and highly squeezed state. An analytical optimization of the cascade, with respect to both the number of particles and the squeezing parameter of each GSS, demonstrates a phase sensitivity

$$
\Delta \theta_{\text {est }}=\frac{4}{N_{T}}
$$
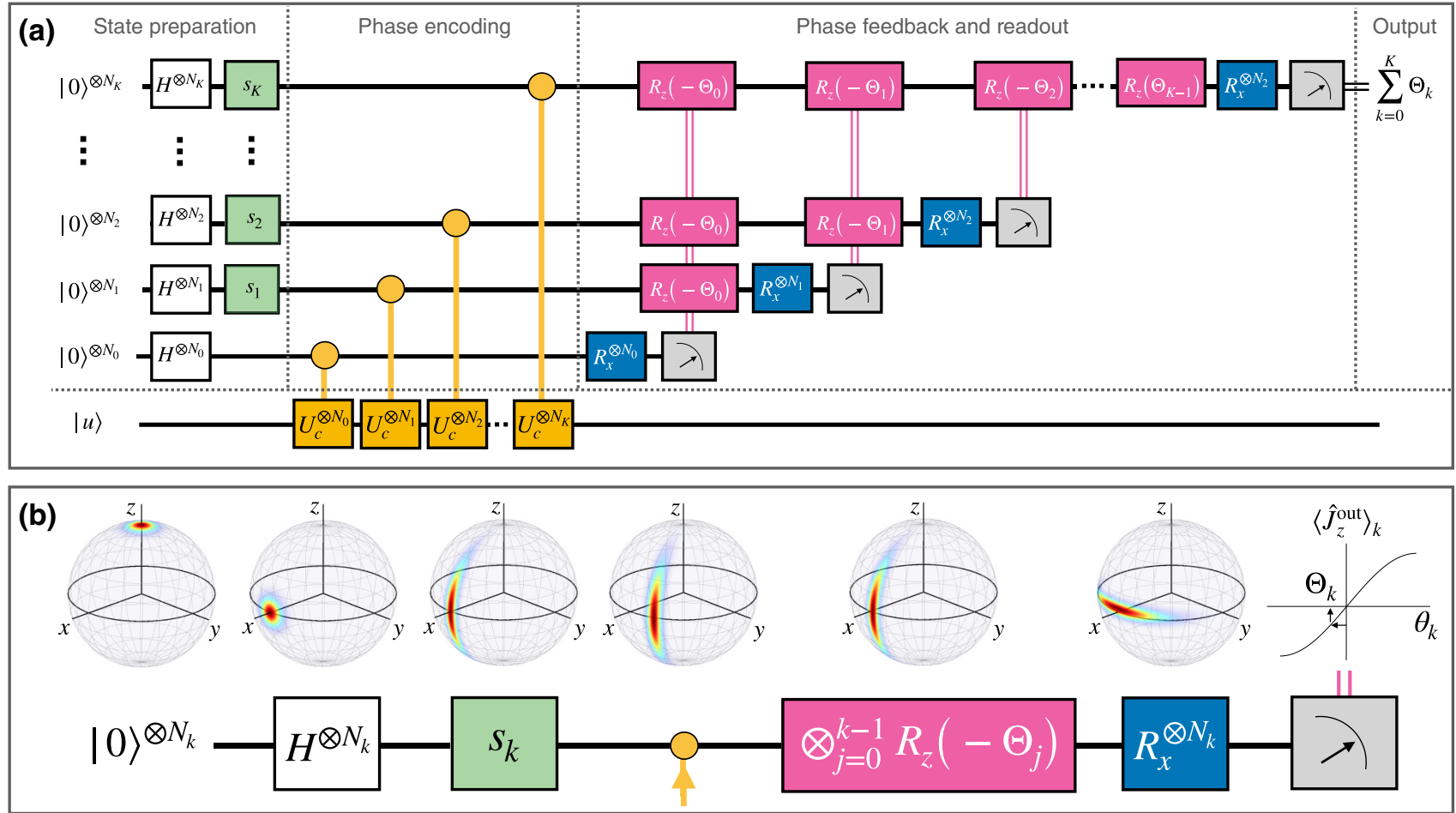

FIG. 1. Circuit diagram of the quantum phase estimation algorithm described in this work. (a) The $K+1$ ancilla states prepared in $|0\rangle^{\otimes N_{k}}(k=0, \ldots, K)$ are first rotated by applying a collective Hadamard gate $H^{\otimes N_{k}}$ (white box). The green box is a spin-squeezing gate, with $s_{k}$ being the squeezing parameter (see the text). The yellow box represents a sequence of $N_{k}$ controlled- $U$ gates, each applied to a single ancilla qubit and providing phase encoding conditioned by the eigenstate $|u\rangle$ of the register: the sequence of controlled- $U$ gates is schematically represented by a single gate indicated as $U_{c}^{\otimes N_{k}}$. The black circle at the end of each vertical yellow line indicates the group of qubits to which the phase encoding is applied. Pink boxes are rotation phase gates $R_{z}\left(-\Theta_{j}\right)$ implementing a classical phase feedback (pink lines) based on the estimate $\Theta_{j}$ at step $j<k$. The readout is obtained by applying a rotation gate $R_{x}^{\otimes N}$ (blue box) followed by a measurement of the eigenstates of $\hat{J}_{z}$ (gray box). The output of the algorithm, after $K+1$ steps, is an estimate, $\theta_{\text {est }}=\sum_{k=0}^{K} \Theta_{k}$, of the unknown phase $\theta$. (b) Illustration of the $k$ th step of the algorithm (bottom) and the corresponding Husimi representation of the quantum state on the $N_{k}$-qubits Bloch sphere (top) for each operation. The output signal $\left\langle\hat{J}_{z}^{\text {out }}\right\rangle_{k}$ has a sinusoidal dependence on $\theta_{k}$ (see the text), from which the estimated $\Theta_{k}$ is extracted and used to phase feedback the next $(k+1)$ step of the algorithm. 
for the estimation of any arbitrary phase $\theta \in[-\pi, \pi)$, where $N_{T}$ is the total number of qubits used in the full estimation algorithm. This result overcomes the performance of other QPE algorithms proposed so far in the literature $[18,23,24]$, including those using GHZ states $[14,15,21-$ 23]. Moreover, our protocol is implemented with a single measurement of a collective spin observable at each step and the phase is extracted with a practical estimation technique.

\section{PHASE ESTIMATION ALGORITHM}

The circuit representation of $K+1$ steps of the algorithm is shown in Fig. 1(a), while Fig. 1(b) illustrates a single step. The phase estimation uses $N_{T}$ particles that can access two internal or spatial modes. The ensemble of $N_{T}$ qubits is divided into $K+1$ spin-polarized states $|0\rangle^{\otimes N_{k}}$, one for each step $k=0,1, \ldots, K$ of the algorithm, with $N_{k} \gg 1$ and $\sum_{k=0}^{K} N_{k}=N_{T}$. We also introduce collective spin operators $\hat{J}_{n}=\sum_{j=1}^{N_{k}} \hat{\sigma}_{n}^{(j)} / 2$, where $\hat{\sigma}_{n}^{(j)}$ is the Pauli matrix of particle $j$ along the axis $n=x, y, z$ in the Bloch sphere. Each state $|0\rangle^{\otimes N_{k}}$ first goes through a collective Hadamard gate $H^{\otimes N_{k}}$, which prepares the coherent spin state $\left|\operatorname{CSS}\left(N_{k}\right)\right\rangle=[(|0\rangle+|1\rangle) / \sqrt{2}]^{\otimes N_{k}}$ aligned along the $x$ axis of the generalized Bloch sphere; see Fig. 1(b). Except at the zeroth step $k=0$, the state $\left|\operatorname{CSS}\left(N_{k}\right)\right\rangle$ then goes through a spin-squeezing gate (represented in Fig. 1 by the green box) that generates the GSS

$$
\left|\psi_{k}\left(N_{k}, s_{k}\right)\right\rangle=\frac{1}{\sqrt{n}} \sum_{\mu=-N_{k} / 2}^{N_{k} / 2} e^{-\mu^{2} /\left(s_{k}^{2} N_{k}\right)}|\mu\rangle_{y} .
$$

This concludes the state preparation. Here, $s_{k}^{2}=4\left(\Delta \hat{J}_{y}\right)_{k}^{2} /$ $N_{k}$ is the squeezing parameter $\left(s_{k}<1\right.$ for metrological spin-squeezed states $[30,31,41]$; see the discussion below), $\left(\Delta \hat{J}_{y}\right)_{k}^{2}=\left\langle\psi_{k}\left|\hat{J}_{y}^{2}\right| \psi_{k}\right\rangle-\left\langle\psi_{k}\left|\hat{J}_{y}\right| \psi_{k}\right\rangle^{2}$ is the variance of $\hat{J}_{y}$ (and analogous definition for the other spin operators), the $|\mu\rangle_{y}$ are eigenstates of $\hat{J}_{y}$ with possible eigenvalues $\mu=$ $-N / 2,-N / 2+1, \ldots, N / 2$, and $n$ provides the normalization. Note that the spin-squeezing gate creates entanglement among the $N_{k}$ ancilla qubits [29,42]. It can implemented in a variety of ways and experimental systems; see Refs. [30,31] for reviews.

The phase encoding is obtained from a controlled- $U$ gate applied to each qubit. The gate gives a phase shift $\theta$ to the qubit in the state $|1\rangle$, while leaving $|0\rangle$ unchanged [35]: more explicitly, $U_{c}|1\rangle|u\rangle=e^{i \theta}|1\rangle|u\rangle$ and $U_{c}|0\rangle|u\rangle=$ $|0\rangle|u\rangle$, where $|u\rangle$ is an eigenstate of $U$ that is stored in the register and $e^{i \theta}$ is the corresponding eigenvalue. In Fig. 1 we follow the conventional representation of the controlled- $U$ gate by a box on the register connected to a single qubit [4]: the corresponding transformation to $N_{k}$ qubits is simplified, for illustration reasons, to a single box that we indicate as $U_{c}^{\otimes N_{k}}$. Overall, the $U_{c}^{\otimes N_{k}}$ operation applied to $\left|\psi_{k}\right\rangle|u\rangle$ (yellow line in Fig. 1) is equivalent to a collective spin rotation of the state $\left|\psi_{k}\right\rangle$ by the angle $\theta$ around the $z$ axis, namely $U_{c}^{\otimes N_{k}}\left|\psi_{k}\right\rangle|u\rangle=$ $e^{-i \theta \hat{J}_{z}}\left|\psi_{k}\right\rangle|u\rangle$ [43]. Note that, in Fig. 1, each horizontal thick black line follows the collective transformation of $N_{k}$ qubits. In principle, there should be $N_{k}$ vertical yellow lines connecting a single-qubit controlled- $U$ gate to each single qubit of the state $\left|\psi_{k}\right\rangle$ : for simplicity, we have represented the transformation $U_{c}^{\otimes N_{k}}$ as a single thick yellow line in the figure. The algorithm requires, in total, $N_{T}$ single-qubit controlled- $U$ gates.

The readout consists of (i) a collective spin rotation of the state around the $z$ axis, implementing the phase feedback (see the discussion below); (ii) a collective $\pi / 2$ rotation around the $x$ axis, $R_{x}^{\otimes N_{k}}=e^{-i \pi \hat{J}_{x} / 2}$; and (iii) a final measurement of $\hat{J}_{z}$, with possible result $-N_{k} / 2 \leq \mu_{k} \leq N_{k} / 2$. At the $k$ th step of the algorithm, the state before the projective measurement is thus $\left|\psi_{k}^{\text {out }}\right\rangle=$ $e^{-i \pi \hat{J}_{x} / 2} e^{-i \theta_{k} \hat{J}_{z}}\left|\psi_{k}\right\rangle$, where $\theta_{k}$ is the overall rotation angle around the $z$ axis, given by the sum of $\theta$ and the phase feedback angle. The average value of $\hat{J}_{z}$ on the output state depends sinusoidally on $\theta_{k}:\left\langle\hat{J}_{z}^{\text {out }}\right\rangle_{k} \equiv\left\langle\psi_{k}^{\text {out }}\left|\hat{J}_{z}\right| \psi_{k}^{\text {out }}\right\rangle=$ $\left\langle\hat{J}_{x}\right\rangle_{k} \sin \theta_{k}$ with $\left\langle\hat{J}_{x}\right\rangle_{k}=\left\langle\psi_{k}\left|\hat{J}_{x}\right| \psi_{k}\right\rangle$. The phase estimation protocol at each step of the QPE algorithm is based on the standard method of moment [30]: replacing $\left\langle\hat{J}_{z}^{\text {out }}\right\rangle$ with the results $\mu_{k}$ of a single measurement of $\hat{J}_{z}$ on the output state leads to the estimate

$$
\Theta_{k}\left(\mu_{k}\right)=\arcsin \frac{\mu_{k}}{\left\langle\hat{J}_{x}\right\rangle_{k}}
$$

of $\theta_{k}$. The sensitivity of the estimator $\Theta_{k}$ is quantified by the mean squared error, $\left(\Delta \Theta_{k}\right)^{2}=\sum_{\mu_{k}=-N_{k} / 2}^{N_{k} / 2} P\left(\mu_{k} \mid \theta_{k}\right)$ $\left[\Theta_{k}\left(\mu_{k}\right)-\theta_{k}\right]^{2}$, where $P\left(\mu_{k} \mid \theta_{k}\right)=\left|\left\langle\psi_{k}^{\text {out }} \mid \mu_{k}\right\rangle\right|^{2}$ is the probability of obtaining the result $\mu_{k}$ for a given $\theta_{k}$. Using error propagation [30], we have $\left(\Delta \Theta_{k}\right)^{2} \approx$ $\left(\Delta \hat{J}_{z}^{\text {out }}\right)_{k}^{2} /\left(d\left\langle\hat{J}_{z}^{\text {out }}\right\rangle_{k} / d \theta_{k}\right)^{2}$, where $\left(\Delta \hat{J}_{z}^{\text {out }}\right)_{k}^{2}=\left\langle\left(\hat{J}_{z}^{\text {out }}\right)^{2}\right\rangle_{k}-$ $\left\langle\hat{J}_{z}^{\text {out }}\right\rangle_{k}^{2}$. This gives

$$
\left(\Delta \Theta_{k}\right)^{2}=\frac{\left(\Delta \hat{J}_{y}\right)_{k}^{2}}{\left\langle\hat{J}_{x}\right\rangle_{k}^{2}}+\frac{\left(\Delta \hat{J}_{x}\right)_{k}^{2}}{\left\langle\hat{J}_{x}\right\rangle_{k}^{2}} \tan ^{2} \theta_{k},
$$

where the $\left(\Delta \hat{J}_{x, y}\right)_{k}^{2}$ are spin variances calculated on the state $\left|\psi_{k}\right\rangle$, and we have taken into account the facts that $\left\langle\hat{J}_{z}\right\rangle_{k}=0$ and $\left\langle\hat{J}_{z} \hat{J}_{x}\right\rangle_{k}=0$ for the GSS Eq. (2). The spin moments appearing in Eq. (4) can be calculate analytically; see Appendix A for details. For $s_{k}^{2} N_{k} \gtrsim 1$, we find $\left\langle\hat{J}_{x}\right\rangle_{k}=N_{k} e^{-1 /\left(2 s_{k}^{2} N_{k}\right)} / 2,\left\langle\hat{J}_{y}\right\rangle_{k}=$ $\left\langle\hat{J}_{z}\right\rangle_{k}=0,\left\langle\hat{J}_{x}^{2}\right\rangle_{k}=N_{k}^{2}\left(1+e^{-2 /\left(s_{k}^{2} N_{k}\right)}\right) / 8,\left\langle\hat{J}_{y}^{2}\right\rangle_{k}=N_{k} s_{k}^{2} / 4$, and $\left\langle\hat{J}_{z}^{2}\right\rangle_{k}=N_{k}^{2}\left(1-e^{-2 /\left(s_{k}^{2} N_{k}\right)}\right) / 8$. The first term in Eq. (4) is the Wineland spin-squeezing parameter [41], $\xi_{R}^{2}=$ $s_{k}^{2} e^{1 / s_{k}^{2} N_{k}}$, corresponding to the phase uncertainty at $\theta_{k}=0$. 
For $s_{k}^{2} N_{k} \gtrsim 1, \xi_{R}^{2}$ decreases with $s_{k}$ and reaches the minimal value $e / N_{k}$ for $s_{k}^{2} N_{k}=1$. The second term in Eq. (4) is due to the bending of the squeezed states in the Block sphere [see, e.g., Fig. 1(b)], $\left(\Delta \hat{J}_{x}\right)_{k}^{2} /\left\langle\hat{J}_{x}\right\rangle_{k}^{2}=$ $2 \sinh ^{2}\left[1 /\left(2 s_{k}^{2} N_{k}\right)\right]$. This term increases when decreasing $s_{k}$ [in particular $\left(\Delta \hat{J}_{x}\right)_{k}^{2} /\left\langle\hat{J}_{x}\right\rangle_{k}^{2} \approx 1 /\left(2 s_{k}^{4} N_{k}^{2}\right)$ for $\left.s_{k}^{2} N_{k} \gg 1\right]$, and is responsible for the strong dependence of $\left(\Delta \Theta_{k}\right)^{2}$ on $\theta_{k}$. This contribution prevents the use of a single (highly) squeezed state for phase estimation: a problem solved here using the adaptive algorithm of Fig. 1.

The key operation of the algorithm is the phase feedback (represented by pink lines and boxes in Fig. 1): after phase encoding, the state $e^{-i \theta \hat{J}_{z}}\left|\psi_{k}\right\rangle$ is sequentially rotated by $e^{i \hat{J}_{z} \Theta_{0}} e^{i \hat{J}_{z} \Theta_{1}} \cdots e^{i \hat{J}_{z} \Theta_{k-1}} \equiv \bigotimes_{j=0}^{k-1} R_{z}\left(-\Theta_{j}\right)$, with $\Theta_{j}$ being the estimated value at the step $j<k$ (depending of the measurement result $\mu_{j}$ ), Eq. (3). In particular, the overall rotation angle of state (2) is $\theta_{k}=\theta-\sum_{j=0}^{k-1} \Theta_{j}$ for $k \geq 1$, and $\theta_{0}=\theta$. The feedback rotation places the GSS $\left|\psi_{k}\right\rangle$ close to its optimal sensitivity point, namely on the equator of the generalized Block sphere; see Fig. 1(b). The estimated value $\Theta_{k}\left(\mu_{k}\right)$ at the $k$ th step is then used to implement the adaptive phase rotation at the $(k+1)$ th step, and so on.

After $K$ steps the circuit outputs are $K+1$ values $\Theta_{0}, \ldots, \Theta_{K}$ : we take their sum, $\theta_{\text {est }}\left(\mu_{0}, \ldots, \mu_{K}\right)=$ $\sum_{k=0}^{K} \Theta_{k}\left(\mu_{k}\right)$, as the estimate of the unknown $\theta$. Note that the circuit described above leads to an estimate of $\theta$ within the inversion region $[-\pi / 2, \pi / 2]$ of the arcsin function, Eq. (3). The algorithm can be extended to the full range $\theta \in[-\pi, \pi)$ by a slight modification of the zeroth step only: in this case, the state $\left|\operatorname{CSS}\left(N_{0}\right)\right\rangle$ is rotated by $e^{-i(\theta / 2) \hat{J}_{z}}$ (instead of $e^{-i \theta \hat{J}_{z}}$ ). The estimator at the zeroth step is now $\Theta_{0}\left(\mu_{0}\right)=2 \arcsin \left(2 \mu_{0} / N_{0}\right) \in[-\pi, \pi)$, which has a sensitivity $\left(\Delta \Theta_{0}\right)^{2}=4 / N_{0}$ : the factor 4 being a direct consequence of the factor 2 dividing the rotation angle. Below, we analyze the sensitivity of the QPE algorithm for the estimation of an arbitrary $\theta \in[-\pi, \pi)$.

\section{PHASE SENSITIVITY}

The sensitivity of the QPE algorithm after $K$ steps is quantified by the mean squared error of $\theta_{\text {est }}$, which we indicate as $\left(\Delta \theta_{\text {est }}\right)^{2}$. As detailed in Appendix $\mathrm{B},\left(\Delta \theta_{\text {est }}\right)^{2}$ is given by

$$
\left(\Delta \theta_{\mathrm{est}}\right)^{2}=\int d \theta_{K} P\left(\theta_{K}\right)\left(\Delta \Theta_{K}\right)^{2}=\mathcal{E}\left[\left(\Delta \Theta_{K}\right)^{2}\right],
$$

where $P\left(\theta_{K}\right)$ is the statistical distribution of $\theta_{K}$ (corresponding to the overall rotation angle in the last step of the algorithm) and $E[\cdot]$ indicates statistical averaging. This equation is qualitatively understood by the fact that $\theta_{\text {est }}-\theta=\sum_{k=0}^{K} \Theta_{k}-\theta=\Theta_{K}-\theta_{K}$ such that the sensitivity of the full QPE algorithm is thus given by the average sensitivity in the estimation of $\theta_{K}$. Indeed, it should be noted that $\theta_{K}$ depends on the stochastic values $\Theta_{j}$ at all previous $j<K$ steps. In other words, $\theta_{K}$ is a random variable and, according to Eq. (5), the sensitivity of the overall QPE algorithms is given by averaging $\left(\Delta \Theta_{K}\right)^{2}$ over all possible values of $\theta_{K}$. As shown in Appendix B, the quantities $\varepsilon\left[\left(\Delta \Theta_{k}\right)^{2}\right]$ for $k=0, \ldots, K$ are related via the recursive relation

$$
\mathscr{E}\left[\left(\Delta \Theta_{k}\right)^{2}\right]=\frac{\left(\Delta \hat{J}_{y}\right)_{k}^{2}}{\left\langle\hat{J}_{x}\right\rangle_{k}^{2}}+\frac{\left(\Delta \hat{J}_{x}\right)_{k}^{2}}{\left\langle\hat{J}_{x}\right\rangle_{k}^{2}} \mathcal{E}\left[\left(\Delta \Theta_{k-1}\right)^{2}\right]
$$

with initial condition $\mathscr{E}\left[\left(\Delta \Theta_{0}\right)^{2}\right]=4 / N_{0}$. Equation (6) can be eventually generalized in the presence of noise by calculating the spin moments for the noisy states, as illustrated below. Replacing the analytical expression for the collective spin moments in Eq. (6), and assuming that $s_{k}^{2} N_{k} \gg 1$ [44], we obtain

$$
\mathcal{E}\left[\left(\Delta \Theta_{k}\right)^{2}\right]=\frac{s_{k}^{2}}{N_{k}}+\frac{\mathscr{E}\left[\left(\Delta \Theta_{k-1}\right)^{2}\right]}{2 s_{k}^{4} N_{k}^{2}} .
$$

First, we minimize Eq. (7) with respect to $s_{k}$, which gives

$$
s_{k}^{2} N_{k}=\left\{N_{k}^{2} \mathcal{E}\left[\left(\Delta \Theta_{k-1}\right)^{2}\right]\right\}^{1 / 3} .
$$

This equation is also understood as a recursive relation giving the squeezing parameters $s_{1}, s_{2}, \ldots, s_{K}$ as a function of $N_{0}, N_{1}, \ldots, N_{K}$, with initial condition $s_{0}=1$ (for the coherent spin state). Using the optimal values of $s_{1}, \ldots, s_{K}$ given in Eq. (8), we have

$$
\begin{gathered}
\min _{s_{1}, \ldots, s_{K}} \mathcal{E}\left[\left(\Delta \Theta_{K}\right)^{2}\right]=\left(\frac{3}{2}\right)^{(3 / 2)\left(1-1 / 3^{K}\right)} \\
\times \frac{4^{1 / 3^{K}}}{N_{K}^{4 / 3} N_{K-1}^{4 / 9} \cdots N_{1}^{4 / 3^{K}} N_{0}^{1 / 3^{K}}}
\end{gathered}
$$

which can now be optimized as a function of $N_{0}, N_{1}, \ldots$, $N_{K}$ with the constraint of a fixed total number of qubits $N_{T}=\sum_{k=0}^{K} N_{k}$. Replacing $N_{0}=N_{T}-\sum_{k=1}^{K} N_{k}$ in Eq. (9) and taking the derivative with respect to $N_{1}, \ldots, N_{K}$ gives a set of $K$ linear equations that can be recast in the matrix form $\left(\mathbf{A}+\mathbf{u u}^{\top}\right) \cdot \mathbf{x}=N_{T} \mathbf{u}$, where $\mathbf{x}=\left(N_{K}, \ldots, N_{1}\right), \mathbf{u}=$ $(1,1, \ldots, 1)$ and

$$
\mathbf{A}=\frac{1}{4}\left(\begin{array}{ccccc}
1 / 3^{K-1} & 0 & 0 & \cdots & 0 \\
0 & 1 / 3^{K-2} & 0 & \cdots & 0 \\
0 & 0 & 1 / 3^{K-3} & \cdots & 0 \\
\vdots & \vdots & \vdots & \ddots & \vdots \\
0 & 0 & 0 & \cdots & 1
\end{array}\right)
$$

The solution of the linear set of equation is found using the Sherman-Morrison formula, giving $\mathbf{x}=\left[N_{T} /\right.$ 
$\left.\left(2 \times 3^{K}-1\right)\right] \mathbf{A}^{-1} \mathbf{u}$. This leads to the optimal number of particles in each state $\left|\psi_{k}\right\rangle$,

$$
N_{k}=4 \times 3^{k-1} N_{0},
$$

and the optimal squeezing parameter

$$
s_{k}^{2}=\frac{3^{5 / 2-(3 / 2)\left(1 / 3^{k-1}\right)-k}}{2^{(5 / 2)\left(1-1 / 3^{k-1}\right)}} \frac{1}{N_{0}^{1-1 / 3^{k}}},
$$

for $k=1, \ldots, K$. In particular, the first step of the protocol is implemented with a GSS containing $N_{1}=4 N_{0}$ particles and, at each further step, the number of particles is increased by a factor $3: N_{k+1}=3 N_{k}$ for $k=1, \ldots, K-1$. The protocol uses GSSs that are more and more squeezed (namely, with $s_{k}$ decreasing with $k$ ) as the number of steps increases, while $s_{k}^{2} N_{k}$ saturates to the asymptotic value $\sqrt{27 / 2}$ for $k \gg 1$. Finally, using Eqs. (9) and (10) and $N_{0}=N_{T} /\left(2 \times 3^{K}-1\right)$ (which is obtained by summing the geometric series $N_{T}=\sum_{k=0}^{K} N_{k}$ ), we obtain the analytically optimized sensitivity

$$
\left(\Delta \theta_{\mathrm{est}}\right)^{2}=\frac{\alpha_{K}^{2}}{N_{T}^{2-1 / 3^{K}}} .
$$

This equation very rapidly (in the number of steps $K$ ) approaches the Heisenberg scaling [45] with respect to the total number of qubits and with a prefactor that converges to $\alpha_{K \rightarrow \infty} \approx 4$. It is also worth noting that the sensitivity is exponential in the number of steps $K \sim \log _{3} N_{T}$, while, with standard QPE protocols, $K \sim \log _{2} N_{T}$ [1,2,18,23]. Already for $K=3$ (which corresponds to a four-step algorithm using one coherent spin state and three spinsqueezed states with decreasing $s_{k}$ ) we obtain a sensitivity $O\left(N_{T}^{-53 / 27}=N_{T}^{-1.96}\right)$, which is very close to the Heisenberg scaling. Further details on the analytical minimization leading to Eq. (12) are reported in Appendix C.

In Fig. 2(a) we show the results of numerical Monte Carlo simulations of our QPE algorithm where $\theta$ is chosen randomly in $[-\pi, \pi)$ with a flat distribution. The sensitivity $\Delta \theta_{\text {est }}$ approaches the Heisenberg scaling, Eq. (1), as a function of the number of qubits (or, equivalently, the number of steps $K+1$ ), independently of the initial value of $N_{0}$. This is confirmed by $N_{T} \times \Delta \theta_{\text {est }}$ approaching the constant value 4 for large $N_{T}$ and different initial $N_{0}$ [colored symbols in Fig. 2(a)]. Here, parameters $s_{k}$ and $N_{k}$ are chosen by a numerical optimization of $\mathcal{E}\left[\left(\Delta \Theta_{k}\right)^{2}\right]$, recursively for $k=1, \ldots, K$. The expected results using Eq. (5) (colored dashed lines, corresponding to dierent values of $N_{0}$ ) agree well with ab-initio numerical simulations of the algorithm (symbols). Circles are obtained for $N_{0}=100$ and after $K=13$ steps of the algorithm. The solid line is $4 / N_{T}$.
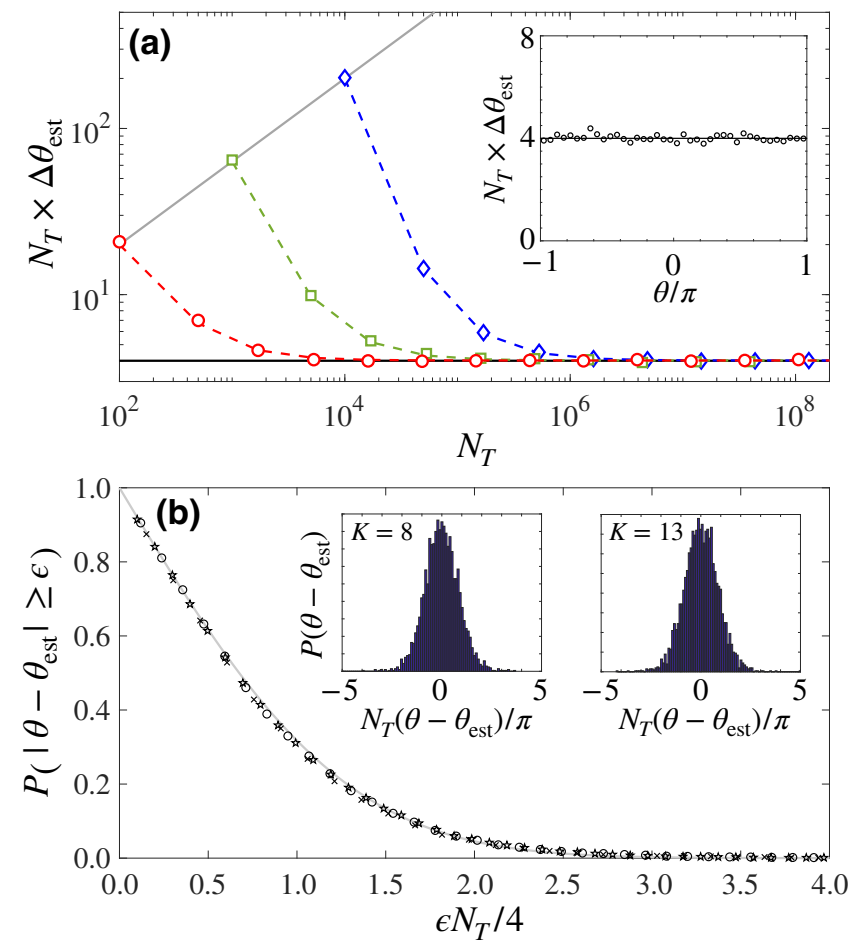

FIG. 2. Phase sensitivity of the QPE algorithm. (a) Phase sensitivity $\Delta \theta_{\text {est }}$ as a function of the total number of particles $N_{T}$ and for different initial values of $N_{0}: N_{0}=10^{2}$ (red lines and symbols), $N_{0}=10^{3}$ (green), and $N_{0}=10^{4}$ (blue). Symbols are results of numerical Monte Carlo simulations where $\theta$ is chosen randomly in $[-\pi, \pi)$. The dotted lines are guides to the eye connecting results of a numerical optimization of Eq. (5) over $s_{k}$ and $N_{k}$ for $k=1, \ldots, K$. The gray line is $2 / \sqrt{N_{T}}$ while the horizontal solid line is $\Delta \theta_{\text {est }}=4 / N_{T}$. The inset shows $\Delta \theta_{\text {est }}$ as a function of $\theta$. Numerical data (circles) correspond to the case $K=13$ and $N_{0}=100$; the solid line is $\Delta \theta_{\text {est }}=4 / N_{T}$. (b) Error probability $P\left(\left|\theta-\theta_{\text {est }}\right| \geq \epsilon\right)$ as a function of $\epsilon N_{T} / 4$. Symbols are Monte Carlo results obtained with $N_{0}=10^{2}$ and $K=8$ (crosses), $K=10$ (stars), $K=13$ (circles). The solid gray line is Eq. (13). The insets show the distributions $P\left(\theta-\theta_{\text {est }}\right)$ for $N_{0}=100$ and different values of $K$. Results in all panels are obtained from $10^{4}$ independent repetitions of the protocol.

In Fig. 2(b) we analyze the behavior of the estimator $\theta_{\text {est }}$. As shown in the insets of Fig. 2(b), the distribution of $\theta-\theta_{\text {est }}$ is - to a very good approximation - a Gaussian centered in 0 , namely the estimator is statistically unbiased (note that the histograms are obtained for $10^{4}$ independent repetitions of the algorithm for random $\theta$ ). Furthermore, the probability of making an error $\epsilon$ in the estimation of an arbitrary $\theta$ is

$$
P\left(\left|\theta_{\mathrm{est}}-\theta\right| \geq \epsilon\right)=1-\operatorname{erf}\left[\epsilon N_{T} /(4 \sqrt{2})\right],
$$

where $\operatorname{erf}(x)$ is the error function; see Fig. 2(b). The error probability is thus exponentially small with $N_{T}$.

So far, we have illustrated the protocol with quantum states having a fixed and known total number of 
particles. In some experimental implementations, for instance with ultracold atoms, it is possible to fix the number of particles only in average, with a fluctuating number of atoms in a single realization. As shown in Appendix $\mathrm{D}$, our protocol is unaffected by these fluctuations. We also note that in some applications, as in atomic clocks $[14,15,49]$, it is necessary to maximize the sensitivity by implementing each round of the protocol with the maximum possible number of particles available, namely $N_{k}=$ $N$ for all $k[50]$.

\section{IMPACT OF DECOHERENCE}

While GSSs are more robust to GHZ states, they are certainly not immune to noise: the aim of this section is to show how the QPE algorithm generalizes in the presence of noise and decoherence. Interestingly, the algorithm can be optimized numerically (and analytically, in some limits) to take into account possible experimental imperfections. As an illustration, we assume the creation of ideal GSSs that are affected by noise before being used in the QPE algorithm and assume noiseless phase rotations (which are typically implemented on time scales much shorter than squeezed-state preparations). We first consider collective dephasing along a specific axis. In this case, the phase sensitivity scaling $\Delta \theta_{\text {est }}=O\left(N_{T}^{-1}\right)$ is also recovered in the presence of strong noise, provided that the GSSs are properly aligned with respect to the dephasing axis. Second, we consider full depolarization of the ideal GSSs. In this case, as a main effect, noise imposes a lower bound on the smallest available squeezing parameter $s$ of the noisy state. This mimics, in practice, the effect of noise in the creation of GSSs in experimental systems.

Collective dephasing along an arbitrary axis $n$ in the Bloch sphere is described by the transformation

$$
\Lambda_{n}\left[\left|\psi_{k}\right\rangle\right]=\int_{-\pi}^{\pi} d \phi P(\phi) e^{-i \phi \hat{J}_{n}}\left|\psi_{k}\right\rangle\left\langle\psi_{k}\right| e^{i \phi \hat{J}_{n}}
$$

This provides a stochastic rotation $e^{-i \phi \hat{J}_{n}}$ with an angle $\phi$ distributed with probability $P(\phi)$, where $\left|\psi_{k}\right\rangle$ is the ideal GSS, Eq. (2). Without loss of generality (upon a further proper rotation of the state $\left.\left|\psi_{k}\right\rangle\right)$ we consider depolarization along the $n=y$ axis. The noise leaves unchanged the moments of $\hat{J}_{y}$ - in particular, it does not affect the squeezing along the $y$ axis - but it decreases the length of the collective spin $\left\langle\hat{J}_{x}\right\rangle$ and increases the bending of the state in the sphere, namely $\left(\Delta \hat{J}_{x}\right)^{2}$. These effects can be taken into account by replacing, in the recursive relation Eq. (6), the spin moments calculated for the noisy state. In particular, the parameters $N_{k}$ and $s_{k}$ can be optimized in the presence of noise (see Appendix D for details). Results of the numerical simulations (symbols) are shown in Figs. 3(a) and $3(\mathrm{~b})$. These are compared with the expected behaviour [dashed lines in Fig. 3(a) and solid line in Fig. 3(b)]. For a
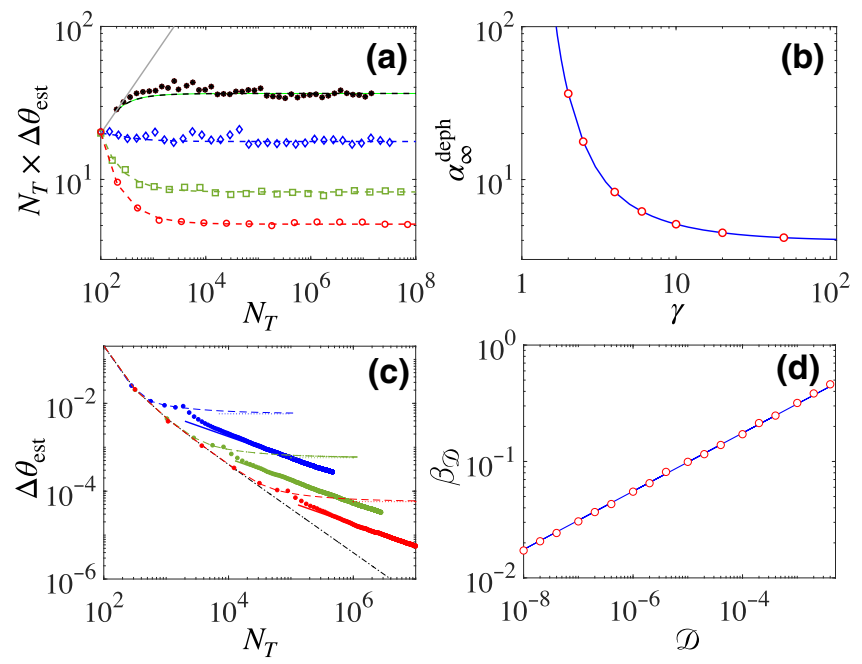

FIG. 3. Phase sensitivity of the QPE in the presence of noise. Panels (a) and (b) consider the effect of collective dephasing described by Eq. (14) with $P(\phi) \propto e^{\gamma \cos \phi}$. Panel (a) reports $\Delta \theta_{\text {est }}$ as a function of the total number of particles $N_{T}$ and for different values of $\gamma: \gamma=10$ (red lines and symbols), 4 (green), 2.5 (blue), and 2 (black). Symbols are results of Monte Carlo simulations, while dotted lines are expected results (see Appendix D). Panel (b) shows the prefactor $\alpha_{\infty}^{\text {deph }}$ of the Heisenberg scaling in Eq. (15) as a function of $\gamma$. Circles are results of Monte Carlo simulations (obtained asymptotically in the number of steps); the solid line is the expected result - see Appendix D. In panels (c) and (d) we consider full depolarization. Panel (c) shows $\Delta \theta_{\text {est }}$ as a function of $N_{T}$ with the symbols representing the results of simulations for different values of $\mathscr{D}: \mathscr{D}=10^{-4}$ (blue circles), $10^{-6}$ (green), and $10^{-8}$ (red). Lines are the analytical results (see the text). The black dashed line is the noiseless case. Asymptotically in $N_{T}$, the sensitivity follows Eq. (17) with prefactor $\beta_{\mathscr{D}}$ shown in panel (d) as a function of $\mathscr{D}$. Here the blue line is $\beta_{\mathscr{D}}=1.75 \times \mathscr{D}^{1 / 4}$, obtained from a fit. Red circles are results of numerical simulations.

sufficiently large number of steps, the protocol reaches the Heisenberg scaling (for $K \gg 1$ )

$$
\Delta \theta_{\mathrm{est}}=\frac{\alpha_{\infty}^{\mathrm{deph}}}{N_{T}},
$$

where the prefactor $\alpha_{\infty}^{\text {deph }}$ is determined by the coefficients $\int_{-\pi}^{\pi} d \phi \cos \left(2^{\lambda} \phi\right) P(\phi)$, with $\lambda=0,1$; see Appendix D. Note that the Heisenberg scaling (15) is recovered even when the width of $P(\phi)$ is of the order of $2 \pi$, highlighting the robustness of the QPE algorithm to this source of noise. This is in contrast with QPE protocols implemented with GHZ states, where there is no preferred rotation axis that guarantees robustness to dephasing noise [51]. On the other hand, if the spin-squeezed state is not properly aligned with respect to the noise axis, noise will impose a lower bound to the squeezing parameter $s$ and the overall effect associated with dephasing will be 
similar to that of full depolarization, which is discussed below.

Full depolarization (within the symmetric subspace of dimension $N+1$ ) is described by the transformation

$$
\Lambda_{\mathscr{D}}\left[\left|\psi_{k}\right\rangle\right]=(1-\mathscr{D})\left|\psi_{k}\right\rangle\left\langle\psi_{k}\right|+\frac{\mathscr{D}}{N+1} \mathbb{1},
$$

where $0 \leq \mathscr{D} \leq 1$ is the depolarization parameter. This noise affects the spin moments along all directions and, in particular, it imposes a lower bound to the smallest achievable squeezing parameter $s^{2} \leq s_{\min }^{2}=\mathscr{D N} / 3$; see Appendix D. Simulations of the QPE in the presence of full depolarization are shown in Figs. 3(c) and 3(d). In general, any noise in the state preparation that limits the preparation of the highly squeezed state, e.g., particle losses (see Appendix D), would have an effect similar to that of full depolarization. The QPE protocol in the presence of noise follows the ideal (noiseless) phase estimation sensitivity up to a number of steps $k \lesssim \tilde{k}$ (corresponding to a number of particles $N_{\tilde{k}} \sim 4 \times 3^{\tilde{k}} N_{0}$ ) for which $s_{k} \geq s_{\min }$. The QPE protocol is robust in the sense that, if $\mathscr{D} \ll 1$ such that $\tilde{k} \gg 1$, we recover the Heisenberg scaling (1) in a finite range of the total number of particles. When $k \geq \tilde{k}$, the sensitivity of the QPE algorithm saturates to the asymptotic value $\Delta \theta_{\text {est }} \cong \sqrt{\mathscr{D} / 3}$; see the dashed lines in Fig. 3(c). This saturation can be avoided by modifying the algorithm: the phase estimation is now repeated with multiple copies of the squeezed state $\left|\psi_{\tilde{k}}\right\rangle$ of $N_{\tilde{k}}$ particles and squeezing parameter $s_{\tilde{k}}$ and without phase feedback between different steps. Asymptotically in $N_{T}$ the algorithm reaches a sensitivity (see Appendix D)

$$
\Delta \theta_{\mathrm{est}}=\frac{\beta_{\mathscr{D}}}{\sqrt{N_{T}}}
$$

with a prefactor $\beta_{\mathscr{D}} \propto \mathscr{D}^{1 / 4}$ that is smaller that 1 (while for separable qubit states, we would have $\Delta \theta_{\text {est }}=1 / \sqrt{N_{T}}$ ). The asymptotic behavior Eq. (17) is in qualitative agreement with the no-go theorem of Refs. $[25,26]$ that prevents reaching the Heisenberg limit for an asymptotically large number of particles and for the noise model of Eq. (16). Symbols in Fig. 3(c) show results of numerical Monte Carlo simulations, in excellent agreement with analytical calculations. In panel (d) we show $\beta_{\mathscr{D}}$ as a function of $\mathscr{D}$ where the results of simulations (circles) are compared to the expected scaling with $\mathscr{D}$ (solid line).

\section{NONLINEAR READOUT AGAINST DETECTION NOISE}

Using a nonlinear readout protocol [52-56], the QPE algorithm can be made robust against detection noise, which is one of the most important sources of noise in atomic experiments targeting entanglement-enhanced
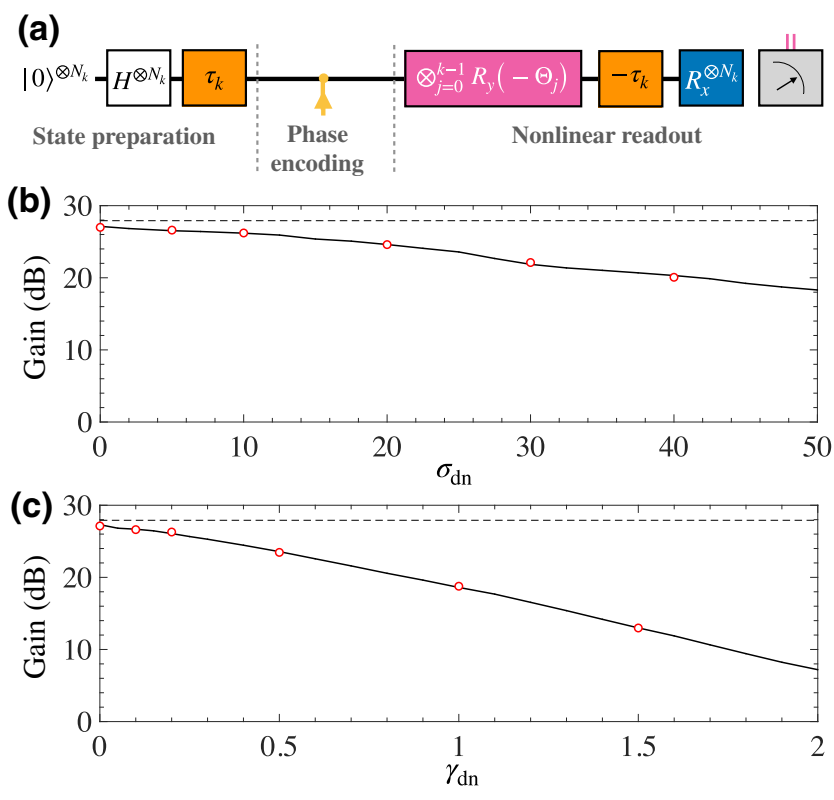

FIG. 4. Phase sensitivity of the QPE in the presence of detection noise. (a) Modified $k$ th step of the QPE algorithm (for $k \geq 1$ ) including the OAT gate (indicated as $\tau_{k}$, orange) and its inverse $\left(-\tau_{k}\right)$; see the text. Panels (b) and (c) show the sensitivity gain in the presence of detection noise. In panel (b), we consider a constant $\sigma_{\mathrm{DN}}$ at all steps (namely independently from the number of particles). In panel (c), we consider $\sigma_{\mathrm{DN}}=\gamma_{\mathrm{DN}} \sqrt{N_{k}}$, which depends on the number of particles at each step. The solid black line is a semianalytical prediction (see Appendix E); the circles are results of numerical simulations for the estimation of a random $\theta \in[-\pi, \pi)$ repeated $10^{4}$ times. The horizontal dashed line corresponds to $\Delta \theta_{\text {est }}=4 / N_{T}$. The vertical axis in panels (b) and (c) shows the gain in decibels with respect to the standard quantum limit, namely $-10 \log _{10}\left[\left(\Delta \theta_{\text {est }}\right)^{2} N_{T}\right]$.

sensitivities. Nonlinear readout has been demonstrated experimentally for atomic squeezed states in Ref. [55]: it consists of a proper nonlinear evolution applied before particle-number detection. In this case, each step of the protocol of Fig. 1 is modified as shown in Fig. 4(a). The spin-squeezing gate is generated by applying a one-axistwisting (OAT) evolution $e^{-i \tau_{k} \hat{J}_{z}^{2}}$ [57] [represented by the orange box indicated as $-\tau_{k}$ in Fig. 4(a)] to an initial coherent spin state, where $\tau_{k}$ is an effective nonlinearevolution time. The readout here consists of four transformations: (i) the phase feedback discussed previously (pink box); (ii) an inverse OAT transformation, $e^{+i \tau_{k} \hat{J}_{z}^{2}}$ (orange box indicated as $-\tau_{k}$ ), (iii) a rotation of $\pi / 2$ around the $x$ axis, $R_{x}^{\otimes N_{k}}=e^{-i \pi \hat{J}_{x} / 2}$ (bluebox), and, finally, (v) a measurement of $\hat{J}_{z}$. Notice that, here, we consider phase encoding and feedback implemented as rotations around the $y$ axis, corresponding to the overall transformation $e^{-i \theta_{k} \hat{J}_{y}}$. This can be implemented as the transformation $e^{-i \theta_{k} \hat{J}_{z}}$, as discussed in Fig. 1, combined with additional rotations around the $x$ axis [not explicitly shown in Fig. 4(a)]: $e^{-i \theta_{k} \hat{J}_{y}}=e^{i(\pi / 2) \hat{J}_{x}} e^{-i \theta_{k} \hat{J}_{z}} e^{-i(\pi / 2) \hat{J}_{x}}$. Precisely, the 
output state before the final projective measurement is now given by

$$
\left|\phi_{k}^{\text {out }}\left(N_{k}, \tau_{k}, \theta_{k}\right)\right\rangle=e^{-i(\pi / 2) \hat{J}_{x}} e^{+i \tau_{k} \hat{J}_{z}^{2}} e^{-i \theta_{k} \hat{J}_{y}}\left|\phi_{k}\left(N_{k}, \tau_{k}\right)\right\rangle,
$$

where $\left|\phi_{k}\left(N_{k}, \tau_{k}\right)\right\rangle=e^{-i \tau_{k} \hat{J}_{z}^{2}}\left|\operatorname{CSS}\left(N_{k}\right)\right\rangle$ is the state after the OAT gate. The rotation angle $\theta_{k}$ is estimated by inverting the relation $\left\langle\hat{J}_{z}^{\text {out }}\right\rangle_{k}=\left\langle\phi_{k}^{\text {out }}\left|\hat{J}_{z}\right| \phi_{k}^{\text {out }}\right\rangle$ as a function of $\theta_{k}$, which is evaluated numerically; see Appendix E.

We have studied the QPE protocol with nonlinear readout. It should be noted that, while the advantage of nonlinear readout has previously been analyzed for the estimation of a phase shift $\theta \approx 0$ [52-56], we extend it here for the estimation of an arbitrary phase $\theta \in[-\pi, \pi)$ with sensitivity robust against detection noise. The protocol is optimized numerically over $N_{k}$ and $\tau_{k}$ at each step (see Appendix E) and, in the noiseless case, it reaches performances analogous to those of the QPE protocol of Fig. 1. The advantage of the nonlinear readout is obtained when including detection noise. We model the noisy detection by the convolution $P\left(\mu_{k} \mid \theta_{k}\right)=\sum_{v=-N_{k} / 2}^{N_{k} / 2} P_{\mathrm{DN}}\left(\mu_{k} \mid v\right) P\left(v \mid \theta_{k}\right)$, where $P\left(v \mid \theta_{k}\right)={ }_{z}\left\langle v\left|\phi_{k}^{\text {out }}\left(N_{k}, \tau_{k}, \theta_{k}\right)\right|^{2}\right.$ is the probability that a measurement of $\hat{J}_{z}$ gives results $v$, and $P_{\mathrm{DN}}\left(\mu_{k} \mid v\right) \approx$ $e^{-(\nu-\mu)^{2} /\left(2 \sigma_{\mathrm{DN}}^{2}\right)}$ is a Gaussian convolution function, where $\sigma_{\mathrm{DN}}$ is the detection noise parameter. In Figs. 4(b) and 4(c) we report the results of numerical simulations of the QPE algorithm showing the sensitivity $\left(\Delta \theta_{\text {est }}\right)^{2}$ as a function of $\sigma_{\mathrm{DN}}$. In particular, in panel (b), $\sigma_{\mathrm{DN}}$ is assumed constant for all steps of the protocol, while in panel (c) we have taken a detection noise that depends on the number of particles $N_{k}$ at each step, namely $\sigma_{\mathrm{DN}}=\gamma_{\mathrm{DN}} \sqrt{N_{k}}$ for the step $k$. In the two panels we plot $\left(\Delta \theta_{\text {est }}\right)^{2}$ for the estimation of an arbitrary phase $\theta \in[-\pi, \pi)$ using an optimized $(K=4)$-step protocol. Numerical simulations (red circles) agree very well with expected results (solid line) and show that a large gain with respect to the standard quantum limit is also possible in the presence of large detection noise (note that in current experiments $\sigma_{\mathrm{DN}}=$ $5 \div 20$ [30], approximately independent from the number of particles).

\section{DISCUSSION AND CONCLUSIONS}

To summarize, our QPE algorithm for the estimation of an arbitrary phase $\theta \in[-\pi, \pi)$ uses Gaussian spin states and an adaptive measurement protocol. It reaches a sensitivity scaling $\Delta \theta_{\text {est }}=O\left(1 / N_{T}\right)$ with respect to the total number of qubits $N_{T}$ or, equivalently, the total number of applications of a controlled- $U$ gate. There are two main differences with respect to the standard QPE algorithms $[1,2]$ using single ancilla qubits [18,24], including those based on the inverse quantum Fourier transform $[3,4,6]$. (i) The number of controlled- $U$ gates in the GSS algorithm of Fig. 1 scales linearly with $N_{T}$ rather than exponentially. This means that, if we take into account the time $t_{U}$ necessary to implement a single controlled- $U$ gate, the GSS algorithm is exponentially faster. This property is also shared by QPE algorithms using GHZ states $[22,23]$ that are however very fragile to noise. The short time required by the algorithm makes it well suited for the estimation of time-varying signals. More explicitly, if we indicate with $\alpha=d \theta(t) / d t$ the local slope of the signal, we obtain the condition $\alpha \lesssim O\left(t_{U} N_{T}^{2}\right)^{-1}$ for the Heisenberg limited estimation of the time varying $\theta$ with the GSS algorithm, compared to the more stringent condition $\alpha \lesssim O\left(t_{U} N_{T} e^{N_{T}}\right)^{-1}$ for the QPE algorithms using single ancilla qubits. (ii) Differently from Kitaev's QPE protocol $[1,2]$, the phase estimation at each step of the GSS algorithm is based on a single measurement. The knowledge about $\theta$ is progressively sharpened using GSSs with a higher and higher number of particles and decreasing squeezing parameters. The key operation of the algorithm is the phase feedback that can be understood as an adaptive measurement $[58,59]$ able to place the GSS around its most sensitive point. Differently from common adaptive strategies $[58,59]$, our protocol uses states of unevenly distributed qubits as a resource for phase estimation. In particular, the analytical optimization provided by Eqs. (10) and (11) allow to fully predetermine the number of particle and squeezing at each step. Therefore, the adaptive measurement in the GSS algorithm does not require the numerical optimization of states, operations, and/or control phases [23,24], nor the support of any classical memory to store the phase distribution [18]. The overall computational time associated with the implementation of the phase feedback in an algorithm of $K$ steps is $(K+1) \times t_{F}$, where $t_{F}$ is the time needed for a single phase feedback, which can be safely assumed to be the same for each step of the protocol. According to Eq. (12), the phase uncertainty becomes exponentially close to the Heisenberg scaling by requiring a linear increase of computational time associated with the phase feedback implementation.

Commonly to all QPE algorithms, our protocol uses controlled- $U$ gates to map a quantity of interest to a phase to be estimated. The GSS algorithm thus shares all known applications of QPE [5,7,8], while using noise-resilient quantum states. The use of GSSs, which are routinely created in labs, can open a novel route for experiments with cold and ultracold atoms toward applications in quantum computing, quantum computational chemistry, and quantum simulation.

\section{ACKNOWLEDGMENTS}

We acknowledge financial support from the European Union's Horizon 2020 research and innovation programme - Qombs Project, FET Flagship on Quantum Technologies Grant No. 820419. 


\section{APPENDIX A: ANALYTICAL CALCULATION OF THE SPIN MOMENTS}

We consider a Gaussian state of $N$ particles

$$
|\psi(N, s)\rangle=\frac{1}{\sqrt{n}} \sum_{\mu=-N / 2}^{N / 2} e^{-\mu^{2} /\left(s^{2} N\right)}|\mu\rangle_{y},
$$

where $s$ is a squeezing parameter $(s<1$ for metrological spin-squeezed states; see below), the $|\mu\rangle_{y}$ are eigenstates of $\hat{J}_{y}\left(\hat{J}_{y}|\mu\rangle_{y}=\mu|\mu\rangle_{y}\right)$, and $n=\sum_{\mu=-N / 2}^{N / 2} e^{-2 \mu^{2} /\left(s^{2} N\right)}$ is the normalization. We calculate mean values and variances of the collective spin operators $\hat{J}_{x, y, z}$ as a function of $N$ and $s$.

\section{First moments}

We have

$$
\begin{aligned}
\left\langle\hat{J}_{x}\right\rangle= & \sum_{\mu=-N / 2}^{N / 2} \frac{e^{-\mu^{2} /\left(s^{2} N\right)} e^{-(\mu+1)^{2} /\left(s^{2} N\right)}}{n} \\
& \times \sqrt{\frac{N}{2}\left(\frac{N}{2}+1\right)-\mu(\mu+1) .}
\end{aligned}
$$

For $s^{2} N \gtrsim 1$, we can replace the sum over $\mu$ with an integral in a continuous variable $m$. For $s \ll \sqrt{N}$, we can neglect boundary effects and extend the integration from $-\infty$ to $+\infty$. We write

$$
\begin{aligned}
\left\langle\hat{J}_{x}\right\rangle \approx & \sqrt{\frac{N}{2}\left(\frac{N}{2}+1\right)} \sqrt{\frac{2}{\pi s^{2} N} \int_{-\infty}^{+\infty} d m e^{-m^{2} /\left(s^{2} N\right)}} \\
& \times e^{-(m+1)^{2} /\left(s^{2} N\right)} \sqrt{1-\frac{m(m+1)}{(N / 2)(N / 2+1)}} .
\end{aligned}
$$

Since the Gaussian wave function has a width of approximately equal to $s^{2} N$, it constraints the variable $m$ to values $|m| \sim s \sqrt{N} \ll N$ : we can thus expand in Taylor the square roots in the above equation to obtain

$$
\begin{aligned}
\left\langle\hat{J}_{x}\right\rangle \approx & \sqrt{\frac{N}{2}\left(\frac{N}{2}+1\right)} \sqrt{\frac{2}{\pi s^{2} N}} \int_{-\infty}^{\infty} d m \\
& \times e^{-m^{2} /\left(s^{2} N\right)} e^{-(m+1)^{2} /\left(s^{2} N\right)} \\
& \times\left[1-\frac{1}{2} \frac{m(m+1)}{(N / 2)(N / 2+1)}\right. \\
& \left.+O\left(\frac{m(m+1)}{(N / 2)(N / 2+1)}\right)^{2}\right] \\
\approx & \sqrt{\frac{N}{2}\left(\frac{N}{2}+1\right)}\left[1-\frac{s^{2} N-1}{(N / 2)(N / 2+1)}\right] \\
& \times e^{-1 /\left(2 s^{2} N\right)} .
\end{aligned}
$$

In particular, in the case $s=1$ we recover the coherent spin state result $\left\langle\hat{J}_{x}\right\rangle=N / 2$ up to corrections of the order of $1 / N$. When $s^{2} N \approx 1$, the discreteness of $\mu$ becomes important and the continuous-variable approximation breaks down. For $s^{2} N \lesssim 1$, the overlap between Gaussians in Eq. (A2) is well approximated by $e^{-\mu^{2} /\left(s^{2} N\right)} e^{-(\mu+1)^{2} /\left(s^{2} N\right)} \approx e^{-1 /\left(s^{2} N\right)}\left(\delta_{\mu, 0}+\delta_{\mu,-1}\right)$, and replacing is into Eq. (A2), we obtain $\left\langle\hat{J}_{x}\right\rangle \approx 2 e^{-1 /\left(s^{2} N\right)}$ $\sqrt{(N / 2)[(N / 2)+1]}$. Overall, keeping only the leading term in $N$, we have

$$
\left\langle\hat{J}_{x}\right\rangle \approx \begin{cases}\frac{N}{2} e^{-1 /\left(2 s^{2} N\right)} & \text { for } s^{2} N \gtrsim 1 \\ N e^{-1 /\left(s^{2} N\right)} & \text { for } s^{2} N \lesssim 1\end{cases}
$$

The other spin moments are equal to zero due to the symmetry properties of the Gaussian state (A1):

$$
\left\langle\hat{J}_{y}\right\rangle=0 \text { and }\left\langle\hat{J}_{z}\right\rangle=0 .
$$

In Fig. 5(a) we plot $\left\langle\hat{J}_{x}\right\rangle$ as a function of $s$. Dots are exact values, the solid line is the analytical approximation for $s^{2} N \gtrsim 1$, while the dashed line is the analytical approximation for $s^{2} N \lesssim 1$.

\section{Second moments}

For the second moment of the $\hat{J}_{x}$ spin operator, we have

$$
\begin{aligned}
\left\langle\hat{J}_{x}^{2}\right\rangle= & \frac{N}{4}+\left(\frac{N^{2}}{8}-\frac{s^{2} N}{8}\right) \\
& +\frac{1}{2} \sum_{\mu=-N / 2}^{N / 2} \frac{e^{-\mu^{2} /\left(s^{2} N\right)} e^{-(\mu+2)^{2} /\left(s^{2} N\right)}}{\eta} \sqrt{\frac{N}{2}+\mu+2} \\
& \times \sqrt{\frac{N}{2}+\mu+1} \sqrt{\frac{N}{2}-\mu \sqrt{\frac{N}{2}-\mu-1}}
\end{aligned}
$$

For $s^{2} N \gtrsim 1$, a continuous-variable approximation and a Taylor expansion yield

$$
\left\langle\hat{J}_{x}^{2}\right\rangle \approx \frac{N}{4}\left(\frac{N}{2}+1\right)\left(1+e^{-2 /\left(s^{2} N\right)}\right)-\frac{s^{2} N}{8} .
$$

We recover the coherent spin state result $\left\langle\hat{J}_{x}^{2}\right\rangle=N^{2} / 4$ for $s=1$ up to $O(1 / N)$. In the opposite limit $s^{2} N \lesssim$ 1 , we consider $e^{-\mu^{2} /\left(s^{2} N\right)} e^{-(\mu+2)^{2} /\left(s^{2} N\right)} \approx e^{-4 /\left(s^{2} N\right)} \delta_{\mu,-2}+$ $e^{-2 /\left(s^{2} N\right)} \delta_{\mu, 1}+e^{-4 /\left(s^{2} N\right)} \delta_{\mu, 0}$ in Eq. (A5) and get

$$
\left\langle\hat{J}_{x}^{2}\right\rangle \approx \frac{N^{2}}{8}\left(1+e^{-2 /\left(s^{2} N\right)}\right)+\frac{N}{4}\left(1-\frac{s^{2}}{2}\right) .
$$

Overall, keeping only the leading term in $N$, we find that

$$
\left\langle\hat{J}_{x}^{2}\right\rangle \approx \frac{N^{2}}{8}\left(1+e^{-2 /\left(s^{2} N\right)}\right)
$$



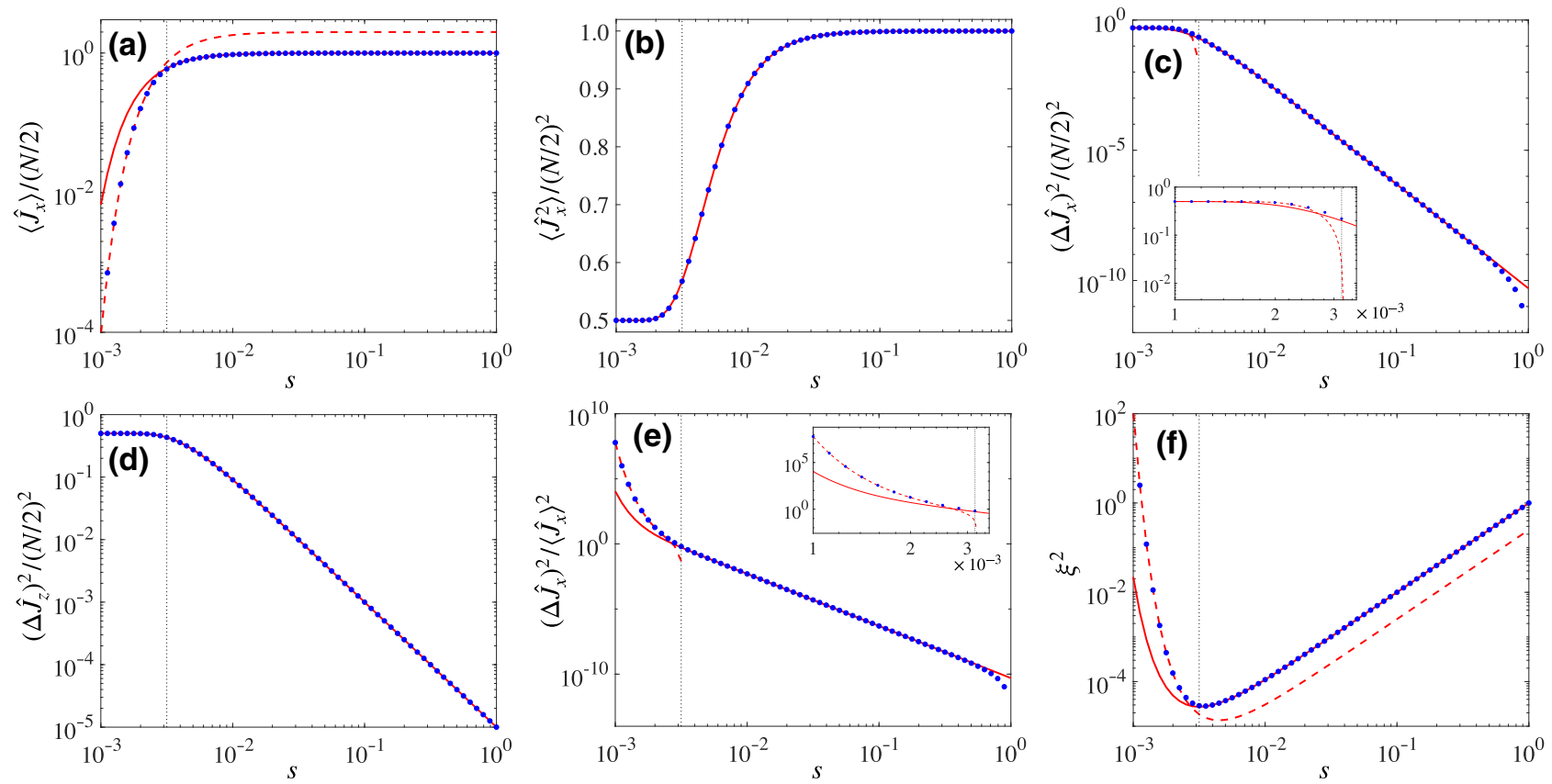

FIG. 5. Spin moments as a function of the squeezing parameter $s$. Dots are exact values and lines are analytical expressions: solid lines are for $s^{2} N \gtrsim 1$ while dashed lines are for $s^{2} N \lesssim 1$. The insets in panels (c) and (e) are enlargements around $s^{2} N=1$. The spin squeezing parameter in panel (f) is $\xi^{2}=N\left(\Delta \hat{J}_{y}\right)^{2} /\left\langle\widehat{J}_{x}\right\rangle^{2}$. In all panels $N=10^{5}$ and the vertical black dotted line is $s^{2} N=1$.

for both $s^{2} N \lesssim 1$ and $s^{2} N \gtrsim 1$. In Fig. 5(b) we plot $\left\langle\hat{J}_{x}^{2}\right\rangle$ as a function of $s$ (dots), compared to the analytical approximation (solid line). The calculation of $\left\langle\hat{J}_{y}^{2}\right\rangle$ is straightforward,

$$
\left\langle\hat{J}_{y}^{2}\right\rangle=\frac{s^{2} N}{4} .
$$

Finally, the calculation of $\left\langle\hat{J}_{z}^{2}\right\rangle$ is similar to the calculation of $\left\langle\hat{J}_{x}^{2}\right\rangle$ and we have

$$
\begin{aligned}
\left\langle\hat{J}_{z}^{2}\right\rangle= & \frac{N}{4}+\left(\frac{N^{2}}{8}-\frac{s^{2} N}{8}\right) \\
& -\frac{1}{2} \sum_{\mu=-N / 2}^{N / 2} \frac{e^{-\mu^{2} /\left(s^{2} N\right)} e^{-(\mu+2)^{2} /\left(s^{2} N\right)}}{n} \sqrt{\frac{N}{2}+\mu+2} \\
& \times \sqrt{\frac{N}{2}+\mu+1} \sqrt{\frac{N}{2}-\mu} \sqrt{\frac{N}{2}-\mu-1}
\end{aligned}
$$

giving

$$
\left\langle\hat{J}_{z}^{2}\right\rangle \approx \frac{N^{2}}{8}\left(1-e^{-2 /\left(s^{2} N\right)}\right)
$$

\section{Variances and covariances}

Combining Eqs. (A3) and (A6) we have

$$
\left(\Delta \hat{J}_{x}\right)^{2} \approx \begin{cases}\frac{N^{2}}{8}\left(1-e^{-1 /\left(s^{2} N\right)}\right)^{2} & \text { for } s^{2} N \gtrsim 1, \\ \frac{N^{2}}{8}\left(1+e^{-2 /\left(s^{2} N\right)}\right)-N^{2} e^{-2 /\left(s^{2} N\right)} & \text { for } s^{2} N \lesssim 1,\end{cases}
$$

while combining Eqs. (A4) and (A7),

$$
\left(\Delta \hat{J}_{y}\right)^{2}=\frac{s^{2} N}{4}
$$

and from Eqs. (A4) and (A8),

$$
\left(\Delta \hat{J}_{z}\right)^{2} \approx \frac{N^{2}}{8}\left(1-e^{-2 /\left(s^{2} N\right)}\right)
$$

for both $s^{2} N \lesssim 1$ and $s^{2} N \gtrsim 1$. In Figs. 5(c) and 5(d), we plot $\left(\Delta \hat{J}_{x}\right)^{2}$ and $\left(\Delta \hat{J}_{z}\right)^{2}$, respectively, as a function of $s$, while in Fig. 5(e) we plot the ratio $\left(\Delta \hat{J}_{x}\right)^{2} /\left\langle\hat{J}_{x}\right\rangle^{2}$. Finally, the covariances are identically zero,

$$
\left\langle\left\{\hat{J}_{x}, \hat{J}_{y}\right\}\right\rangle=0, \quad\left\langle\left\{\hat{J}_{x}, \hat{J}_{z}\right\}\right\rangle=0, \quad \text { and } \quad\left\langle\left\{\hat{J}_{y}, \hat{J}_{z}\right\}\right\rangle=0 \text {, }
$$

for both $s^{2} N \lesssim 1$ and $s^{2} N \gtrsim 1$, to the leading order in $N . \quad$ due to the symmetry of the state (A1). 


\section{Spin-squeezing parameter}

The metrological spin-squeezing parameter is obtained by taking the ratio between Eqs. (A10) and (A3):

$$
\xi^{2}=\frac{N\left(\Delta \hat{J}_{y}\right)^{2}}{\left\langle\hat{J}_{x}\right\rangle^{2}}= \begin{cases}s^{2} e^{1 /\left(s^{2} N\right)} & \text { for } s^{2} N \gtrsim 1, \\ \frac{s^{2}}{4} e^{2 /\left(s^{2} N\right)} & \text { for } s^{2} N \lesssim 1 .\end{cases}
$$

The function $s^{2} e^{1 /\left(s^{2} N\right)}$ reaches a minimum at $s^{2} N=1$. Therefore, for $1 / N \lesssim s^{2}<1$, the spin-squeezing parameter is always decreasing with $s$, reaching a minimum value $\xi_{\min }^{2}=e / N$. For $s^{2} N \lesssim 1$, the exponential term in Eq. (A13) dominates and the spin-squeezing parameter increases with $s$ : in other words, the decrease of $\left\langle\hat{J}_{x}\right\rangle$ with $s$ dominates over the squeezing of $\left(\Delta \hat{J}_{y}\right)^{2}$. In Fig. 5(f) we compare the above approximate expressions with the exact calculation of $\xi^{2}$, as a function of the squeezing parameter $s$.

\section{APPENDIX B: DERIVATION OF EQS. (5) AND (6)}

The sensitivity of the estimator $\theta_{\text {est }}\left(\mu_{0}, \ldots, \mu_{K}\right)=$ $\sum_{k=0}^{K} \Theta_{k}\left(\mu_{k}\right)$ is formally given by

$\left(\Delta \theta_{\mathrm{est}}\right)^{2}=\sum_{\mu_{0}, \ldots, \mu_{K}} P\left(\mu_{0}, \ldots, \mu_{K} \mid \theta\right)\left[\theta_{\mathrm{est}}\left(\mu_{0}, \ldots, \mu_{K}\right)-\theta\right]^{2}$,

where $\mu_{k}$ is the measurement result at the $k$ th step of the algorithm (with $k=0,1, \ldots, K$ ) and the sum runs over all possible measurement results at all steps. The different measurement events are independent and, taking into account the frequency feedback, we can write

$$
\begin{aligned}
P\left(\mu_{0}, \ldots, \mu_{K} \mid \theta\right)= & P\left(\mu_{0}, \ldots, \mu_{K-1} \mid \theta\right) \\
& \times P\left[\mu_{K} \mid \theta_{K}\left(\mu_{0}, \ldots, \mu_{K-1}\right)\right],
\end{aligned}
$$

where $\quad \theta_{K}\left(\mu_{0}, \ldots, \mu_{K-1}\right)=\theta-\sum_{k=0}^{K-1} \Theta_{k}\left(\mu_{k}\right) . \quad$ As noted in the main text, $\theta_{\text {est }}\left(\mu_{0}, \ldots, \mu_{K}\right)-\theta=\Theta_{K}$
$\left(\mu_{K}\right)-\left[\theta-\sum_{k=0}^{K-1} \Theta_{k}\left(\mu_{k}\right)\right]=\Theta_{K}\left(\mu_{K}\right)-\theta_{K}\left(\mu_{0}, \ldots\right.$, $\mu_{K-1}$ ). Equation (B1) can thus be rewritten as

$$
\begin{aligned}
\left(\Delta \theta_{\mathrm{est}}\right)^{2}= & \sum_{\mu_{0}, \ldots, \mu_{K-1}} P\left(\mu_{0}, \ldots, \mu_{K-1} \mid \theta\right) \\
& \times \sum_{\mu_{K}} P\left[\mu_{K} \mid \theta_{K}\left(\mu_{0}, \ldots, \mu_{K-1}\right)\right] \\
& \times\left[\Theta_{K}\left(\mu_{K}\right)-\theta_{K}\left(\mu_{0}, \ldots, \mu_{K-1}\right)\right]^{2} .
\end{aligned}
$$

As a consequence of the stochastic nature of the measurement events, the phase $\theta_{K}\left(\mu_{0}, \ldots, \mu_{K-1}\right)$ is also a stochastic variable with distribution

$$
\begin{aligned}
P\left(\theta_{K}\right)= & \sum_{\mu_{0}, \ldots, \mu_{K-1}} P\left(\mu_{0}, \ldots, \mu_{K-1} \mid \theta\right) \\
& \times \delta\left[\theta_{K}\left(\mu_{0}, \ldots, \mu_{K-1}\right)-\theta_{K}\right],
\end{aligned}
$$

where $\delta$ is the Dirac delta function. Using Eq. (B3), we can thus rewrite Eq. (B2) as

$$
\begin{aligned}
\left(\Delta \theta_{\mathrm{est}}\right)^{2} & =\int d \theta_{K} P\left(\theta_{K}\right) \sum_{\mu_{K}} P\left(\mu_{K} \mid \theta_{K}\right)\left[\Theta_{K}\left(\mu_{K}\right)-\theta_{K}\right]^{2} \\
& =\int d \theta_{K} P\left(\theta_{K}\right)\left(\Delta \Theta_{K}\right)^{2} \\
& \equiv \mathcal{E}\left[\left(\Delta \Theta_{K}\right)^{2}\right]
\end{aligned}
$$

which recovers Eq. (5) of the main text. Next, we substitute Eq. (4) into Eq. (B4) and note that, since we expect $\theta_{K} \ll 1$, we can approximate $\tan ^{2} \theta_{K} \approx \theta_{K}^{2}$. We thus have

$$
\mathcal{E}\left[\left(\Delta \Theta_{K}\right)^{2}\right]=\frac{\left(\Delta \hat{J}_{y}\right)_{K}^{2}}{\left\langle\hat{J}_{x}\right\rangle_{K}^{2}}+\frac{\left(\Delta \hat{J}_{x}\right)_{K}^{2}}{\left\langle\hat{J}_{x}\right\rangle_{K}^{2}} \int d \theta_{K} P\left(\theta_{K}\right) \theta_{K}^{2} .
$$

Note that $\theta_{k}\left(\mu_{0}, \ldots, \mu_{K-1}\right)=\theta-\sum_{k=0}^{K-1} \Theta_{k}\left(\mu_{k}\right)=\theta_{K-1}$ $\left(\mu_{0}, \ldots, \mu_{K-2}\right)-\Theta_{K-1}\left(\mu_{K-1}\right)$ and, thus, using Eq. (B3),

$$
\begin{aligned}
\int d \theta_{K} P\left(\theta_{K}\right) \theta_{K}^{2}= & \sum_{\mu_{0}, \ldots, \mu_{K-1}} P\left(\mu_{0}, \ldots, \mu_{K-1} \mid \theta\right)\left[\theta_{K-1}\left(\mu_{0}, \ldots, \mu_{K-2}\right)-\Theta_{K-1}\left(\mu_{K-1}\right)\right]^{2} \\
= & \sum_{\mu_{0}, \ldots, \mu_{K-2}} P\left(\mu_{0}, \ldots, \mu_{K-2} \mid \theta\right) \sum_{\mu_{K-1}} P\left[\mu_{K-1} \mid \theta_{K-1}\left(\mu_{0}, \ldots, \mu_{K-2}\right)\right] \\
& \times\left[\theta_{K-1}\left(\mu_{0}, \ldots, \mu_{K-2}\right)-\Theta_{K-1}\left(\mu_{K-1}\right)\right]^{2} .
\end{aligned}
$$

Analogously as above, we can understand $\theta_{K-1}\left(\mu_{0}, \ldots, \mu_{K-2}\right)$ as a stochastic variable with distribution

$$
P\left(\theta_{K-1}\right)=\sum_{\mu_{0}, \ldots, \mu_{K-2}} P\left(\mu_{0}, \ldots, \mu_{K-2} \mid \theta\right) \delta\left[\theta_{K}\left(\mu_{0}, \ldots, \mu_{K-2}\right)-\theta_{K-1}\right]
$$


and write

$$
\begin{aligned}
\int & d \theta_{K} P\left(\theta_{K}\right) \theta_{K}^{2} \\
= & \int d \theta_{K-1} P\left(\theta_{K-1}\right) \\
& \times \sum_{\mu_{K-1}} P\left(\mu_{K-1} \mid \theta_{K-1}\right)\left[\theta_{K-1}-\Theta_{K-1}\left(\mu_{K-1}\right)\right]^{2} \\
= & \int d \theta_{K-1} P\left(\theta_{K-1}\right)\left(\Delta \Theta_{K-1}\right)^{2} \\
\equiv & \mathcal{E}\left[\left(\Delta \Theta_{K-1}\right)^{2}\right] .
\end{aligned}
$$

Combining Eqs. (B5) and (B6), we have thus demonstrated that

$$
\mathcal{E}\left[\left(\Delta \Theta_{K}\right)^{2}\right]=\frac{\left(\Delta \hat{J}_{y}\right)_{K}^{2}}{\left\langle\hat{J}_{x}\right\rangle_{K}^{2}}+\frac{\left(\Delta \hat{J}_{x}\right)_{K}^{2}}{\left\langle\hat{J}_{x}\right\rangle_{K}^{2}} \mathcal{E}\left[\left(\Delta \Theta_{K-1}\right)^{2}\right] .
$$

Furthermore, we can write

$$
\begin{aligned}
\mathscr{E}\left[\left(\Delta \Theta_{K-1}\right)^{2}\right]= & \frac{\left(\Delta \hat{J}_{y}\right)_{K-1}^{2}}{\left\langle\hat{J}_{x}\right\rangle_{K-1}^{2}}+\frac{\left(\Delta \hat{J}_{x}\right)_{K-1}^{2}}{\left\langle\hat{J}_{x}\right\rangle_{K-1}^{2}} \\
& \times \int d \theta_{K-1} P\left(\theta_{K-1}\right) \theta_{K-1}^{2},
\end{aligned}
$$

and repeat the above reasoning to obtain the recursive formula (6). It should be noted that in the zeroth step of the protocol, the phase is fixed (and not random), equal to $\theta$ : $\mathcal{E}\left[\left(\Delta \Theta_{0}\right)^{2}\right]=\left(\Delta \Theta_{0}\right)^{2}=4 / N_{0}$, which provides the initial condition for the recursive formula (6).

\section{APPENDIX C: DETAILS ON THE NUMERICAL AND ANALYTICAL MINIMIZATIONS}

When substituting the analytical expressions for the average spin operators and variances discussed above, Eqs. (A3), (A9) and (A10), for $s^{2} N \gtrsim 1$, into Eq. (6), we obtain

$$
\mathcal{E}\left[\left(\Delta \Theta_{k}\right)^{2}\right]=\frac{2 s_{k}^{2}+N_{k}\left(1-e^{-1 /\left(s_{k}^{2} N_{k}\right)}\right)^{2} \mathscr{E}\left[\left(\Delta \Theta_{k-1}\right)^{2}\right]}{2 N_{k} e^{-1 /\left(s_{k}^{2} N_{k}\right)}} .
$$

We recover Eq. (7) when taking the leading order in $s_{k}^{2} N_{k} \gg 1$. The optimization of Eq. (C1) over $s_{k}$ leads to

$$
s_{k}^{4} N_{k}^{2}-s_{k}^{2} N_{k}+N_{k}^{2}\left(\frac{e^{-2 /\left(s_{k}^{2} N_{k}\right)}-1}{2}\right) \mathcal{E}\left[\left(\Delta \Theta_{k-1}\right)^{2}\right]=0,
$$

which can be solved analytically in the limit $s_{k}^{2} N_{k} \gg 1$, giving Eq. (8). By replacing Eq. (8) into Eq. (7) gives

$$
\min _{s_{k}} \mathscr{E}\left[\left(\Delta \Theta_{k}\right)^{2}\right]=\frac{3}{2}\left(\frac{\mathscr{E}\left[\left(\Delta \Theta_{k-1}\right)^{2}\right]}{N_{k}^{4}}\right)^{1 / 3} .
$$

Recursively to $K$ steps of the algorithm, we obtain

$$
\begin{aligned}
\min _{s_{1}, \ldots, s_{K}} \mathcal{E}\left[\left(\Delta \Theta_{K}\right)^{2}\right]= & \left(\frac{3}{2}\right)^{1+1 / 3+1 / 9+\cdots+1 / 3^{K-1}} \\
& \times N_{K}^{-4 / 3} N_{K-1}^{-4 / 9} \cdots N_{1}^{-4 / 3^{K}}\left(\frac{N_{0}}{4}\right)^{-1 / 3^{K}} .
\end{aligned}
$$

The above equation is optimized with respect to $s_{k}$ for $k=1, \ldots, K$ (with $s_{0}$ the initial condition). We now optimize it with respect to $N_{k}$ for $k=$ $0, \ldots, K$, with the constraint of a total $N_{T}=\sum_{k=0}^{K} N_{k}$ particles. Imposing $\left(\partial / \partial N_{k}\right)\left\{1 / \min _{s_{1}, \ldots, s_{K}} \mathcal{E}\left[\left(\Delta \Theta_{k}\right)^{2}\right]\right\}=0$ (with $k=1, \ldots, K$ ) provides the following set of $K$ coupled linear equations:

$$
\begin{gathered}
\left(\frac{1}{4 \times 3^{K-1}}+1\right) N_{K}+N_{K-1}+N_{K-2}+\cdots+N_{1}=N_{T}, \\
N_{K}+\left(\frac{1}{4 \times 3^{K-2}}+1\right) N_{n-1}+N_{K-2}+\cdots+N_{1}=N_{T}, \\
N_{K}+N_{K-1}+\left(\frac{1}{4 \times 3^{K-3}}+1\right) N_{K-2}+\cdots+N_{1}=N_{T}, \\
\vdots \\
N_{K}+N_{K-1}+N_{K-2}+\cdots+\left(1+\frac{1}{4}\right) N_{1}=N_{T} .
\end{gathered}
$$

These can be recast in matrix form as

$$
\left(\mathbf{A}+\mathbf{u} \mathbf{u}^{\top}\right) \cdot \mathbf{x}=N_{T} \mathbf{u},
$$

where $\mathbf{x}=\left(N_{K}, \ldots, N_{1}\right), \mathbf{u}=(1,1, \ldots, 1)$, and $\mathbf{A}$ is given by

$$
\mathbf{A}=\frac{1}{4}\left(\begin{array}{ccccc}
1 / 3^{K-1} & 0 & 0 & \cdots & 0 \\
0 & 1 / 3^{K-2} & 0 & \cdots & 0 \\
0 & 0 & 1 / 3^{K-3} & \cdots & 0 \\
\vdots & \vdots & \vdots & \ddots & \vdots \\
0 & 0 & 0 & \cdots & 1
\end{array}\right) .
$$

The matrix $\mathbf{A}+\mathbf{u u}^{\top}$ can be inverted using the ShermanMorrison formula,

$$
\left(\mathbf{A}+\mathbf{u u}^{\top}\right)^{-1}=\mathbf{A}^{-1}-\frac{\mathbf{A}^{-1} \mathbf{u u}^{\top} \mathbf{A}^{-1}}{1+\mathbf{u}^{\top} \mathbf{A}^{-1} \mathbf{u}}
$$

where we note that

$$
\mathbf{u}^{\top} \mathbf{A}^{-1} \mathbf{u}=\operatorname{Tr}\left[\mathbf{A}^{-1}\right]=4 \times 3^{K} \sum_{k=1}^{K} \frac{1}{3^{k}}=2\left(3^{K}-1\right) .
$$


Therefore,

$$
\left(\mathbf{A}+\mathbf{u} \mathbf{u}^{\top}\right)^{-1} \mathbf{u}=\frac{\mathbf{A}^{-1} \mathbf{u}}{1+\operatorname{Tr}\left[\mathbf{A}^{-1}\right]} .
$$

Finally,

$$
\mathbf{x}=\frac{N_{T}}{2 \times 3^{K}-1} \mathbf{A}^{-1} \mathbf{u}
$$

that is,

$$
\begin{gathered}
N_{K}=\frac{4 \times 3^{K-1}}{2 \times 3^{K}-1} N_{T}=\frac{4}{3} \times \frac{N_{T}}{2-1 / 3^{K}}, \\
N_{K-1}=\frac{4 \times 3^{K-2}}{2 \times 3^{K}-1} N_{T}=\frac{4}{9} \times \frac{N_{T}}{2-1 / 3^{K}}, \\
\vdots \\
N_{k}=\frac{4 \times 3^{k-1}}{2 \times 3^{K}-1} N_{T}=3^{k-1} \times \frac{4}{3^{K}} \times \frac{N_{T}}{2-1 / 3^{K}}, \\
\vdots \\
N_{1}=\frac{4}{2 \times 3^{K}-1} N_{T}=\frac{4}{3^{K}} \times \frac{N_{T}}{2-1 / 3^{K}},
\end{gathered}
$$

and

$$
N_{0}=N_{T}-\sum_{k=1}^{K} N_{k}=\frac{1}{3^{K}} \times \frac{N_{T}}{2-1 / 3^{K}} .
$$

These results are summarized by Eq. (10). In particular, we have $N_{1}=4 N_{0}$ and $N_{k+1}=3 N_{k}$ for $k=1, \ldots, K-1$. Finally, by substituting these values into Eq. (C3), we obtain Eq. (12), where

$$
\alpha_{K}=\frac{3^{(9 / 4)\left(1-1 / 3^{K}\right)-K /\left(2 \times 3^{K}\right)}}{2^{(11 / 4)\left(1-1 / 3^{K}\right)-1 / 3^{K}}}\left(2-\frac{1}{3^{K}}\right)^{1-1 /\left(2 \times 3^{K}\right)} .
$$

Equation (12) reaches a scaling of sensitivity with $N_{T}$ that is arbitrarily close to the Heisenberg scaling when increasing the number of steps of the protocol. In particular, $\lim _{K \rightarrow \infty} \alpha_{K}=3^{9 / 4} / 2^{7 / 4} \approx 3.52$.

\section{Pseudocode of the algorithm}

For further clarity, we report below the pseudocode of the algorithm, according to the analytical optimization discussed above. Note that, as initial conditions, we can either fix the total number of qubits, $N_{T}$, or the number of qubits in the zeroth-step state, $N_{0}$. In the former case, the code starts with $N_{0}=N_{T} /\left(2 \times 3^{K}-1\right)$, whereas in the latter case, it uses a total of $N_{T}=\left(2 \times 3^{K}-1\right) N_{0}$ qubits. We always assume that $N_{0} \gg 1$. Given $N_{T}$ qubits, one can further optimize the algorithm over the number of steps $K$ (see below).

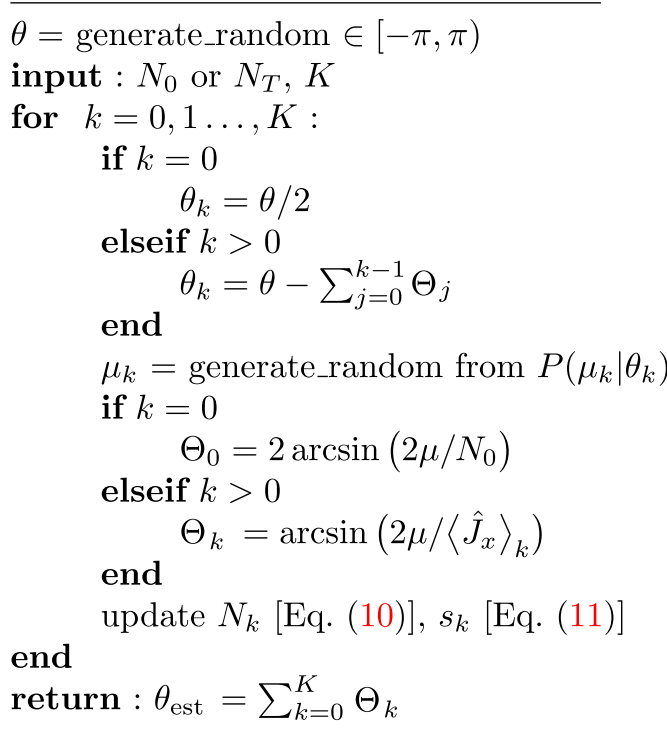

The analytical optimization over $N_{k}$ and $s_{k}$, Eqs. (10) and (11), respectively, relies on the condition $s_{k}^{2} N_{k} \gg 1$ : when this condition is not met, we perform a numerical optimization of Eq. (C1) over $N_{k}$ and $s_{k}$, recursively. Numerical Monte Carlo simulations of the QPE algorithm require the probability distribution $P\left(\mu_{k} \mid \theta_{k}\right)$ in order to simulate stochastic measurement events. It can be calculated exactly as $P\left(\mu_{k} \mid \theta_{k}\right)=\left.\right|_{z}\langle\mu| e^{-i(\pi / 2) \hat{J}_{x}} e^{-i \theta_{k} \hat{J}_{z}}$ $\left.\left|\psi_{k}\right\rangle\right|^{2}$, up to $N_{k} \approx 10^{4}$. For larger values of $N_{k}$, we approximate $P\left(\mu_{k} \mid \theta_{k}\right)$ as a Gaussian distribution centered in $\left\langle\hat{J}_{z}^{\text {out }}\right\rangle_{k}$ with width $\left(\Delta \hat{J}_{z}^{\text {out }}\right)_{k}^{2}$, where $\hat{J}_{z}^{\text {out }}$ can be expressed as a function of the spin moments of the states after state preparation as $\hat{J}_{z}^{\text {out }}=\hat{J}_{x} \sin \theta_{k}+\hat{J}_{z} \cos \theta_{k}$. We confirm that the two approaches give equivalent results for small values of $N_{k}$.

\section{Results for a fixed number of steps}

In Fig. 6 we show the sensitivity as a function of $N_{T}$ for the estimation of a random phase in $[-\pi, \pi]$ and a fixed total number of steps: $K=1$ (black circles and lines), $K=2$ (blue), $K=3$ (green), and $K=4$ (red). Circles are results of numerical Monte Carlo simulations. The solid lines are solutions of a numerical optimization over $s_{k}$ and $N_{k}$ for $k=0, \ldots, K$ (with $s_{0}=1$ ). The dashed line is Eq. (12). The agreement between the numerical results and the analytical prediction relies on the condition $s_{k}^{2} N_{k} \gg 1$ for all $k=0, \ldots, K$. Using Eq. (12), we obtain

$$
s_{K}^{2} N_{K} \sim N_{T}^{1 / 3^{K}}
$$

We thus obtain $s_{k}^{2} N_{k} \gg 1$ for large $N_{T}$ : when increasing $K$, increasingly larger values of $N_{T}$ are required in order to fulfill the condition $s_{k}^{2} N_{k} \gg 1$. This expected behavior is supported by the numerical calculations shown in Fig. (6). 


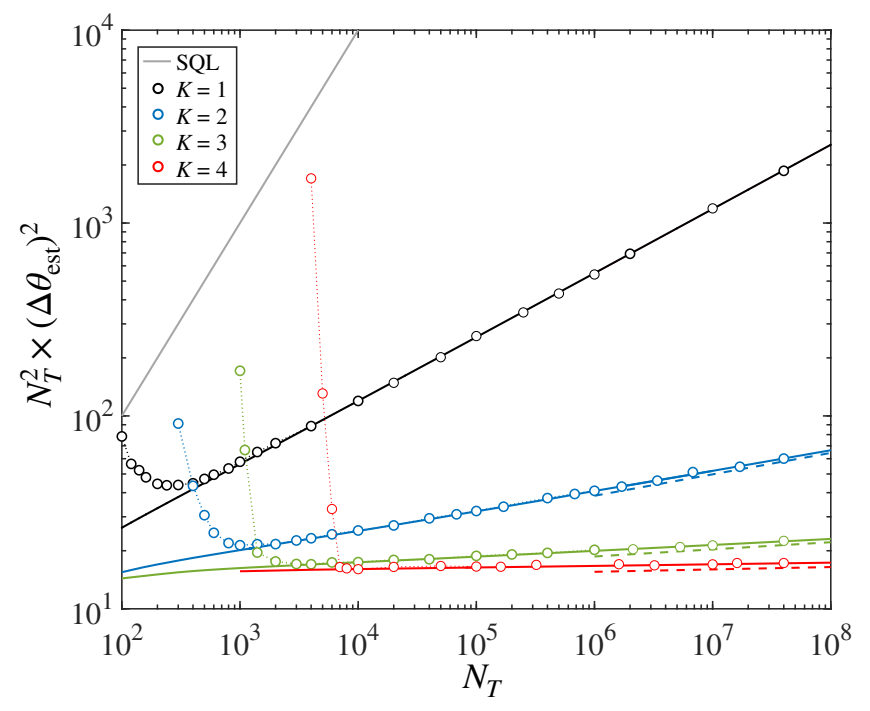

FIG. 6. Phase sensitivity for a fixed number of steps. Plot of $\Delta^{2} \theta_{\text {est }}$ multiplied by $N_{T}^{2}$ as a function of the total number of particles $N_{T}$ for $K$-step protocols: $K=1$ (black), $K=2$ (blue), $K=3$ (green), and $K=4$ (red). Dots are the results of Monte Carlo simulations taking $\theta$ as a random phase in $[-\pi, \pi]$. The solid lines are expected sensitivities (obtained from numerical optimization), while the dashed lines are the analytical predictions, Eq. (12), which hold in the large- $N_{T}$ limit. Dotted lines are guides to the eye. The solid gray line is the standard quantum limit $(\mathrm{SQL})(\Delta \theta)_{\mathrm{SQL}}^{2}=1 / N_{T}$.

This figure also shows that, for a given $N_{T}$, there is an optimal number of steps $K$ that optimizes the sensitivity. For instance, at $N_{T}=1000$, the case $K=2$ overcomes both the cases $K=1$ and $K=3$. The two-step protocol reaches an optimal point at $N_{T} \approx 1000$, where $N_{T} \Delta^{2} \theta_{\text {est }, K}=4.64$.

\section{Heisenberg scaling}

Given a fixed $N_{0}$ (which here plays the role of an initial condition) and asymptotically large $N_{T}$ and $K$, Eq. (12) predicts a sensitivity

$$
\Delta \theta_{\text {est }}=\frac{3.52}{N_{T}}
$$

in the estimation of any $\theta \in[0,2 \pi]$. This result relies on the condition $s_{k}^{2} N_{k} \gg 1$ for all $k=0, \ldots, K$. The quantity $s_{k}^{2} N_{k}$ can be written as a function of $N_{0}$ as

$$
s_{k}^{2} N_{k}=\frac{3^{(3 / 2)\left(1-1 / 3^{k-1}\right)}}{2^{(5 / 2)\left(1-1 / 3^{k-1}\right)-2}} N_{0}^{1 / 3^{k}}
$$

where we have used Eqs. (8), (10) and (12). The value of $N_{0}$ is negligible in Eq. (C7) for large $k$. Asymptotically in $k$, we find that

$$
\lim _{k \rightarrow \infty} s_{k}^{2} N_{k}=\sqrt{\frac{3^{3}}{2}} \approx 3.67 .
$$

We thus conclude that the condition $s_{k}^{2} N_{k} \gg 1$ is not satisfied for large $k$. We can improve the analytical prediction of Eq. (C6) is different ways [see Fig. 7(a) for a comparison between the different methods].

(a) Heuristically (without a rigorous justification), we can take into account the exponential in the denominator of Eq. (C1) by writing

$$
\Delta \theta_{\mathrm{est}}=\frac{\tilde{\alpha}_{K}}{N_{T}^{1-1 /\left(2 \times 3^{K}\right)}},
$$

where $\tilde{\alpha}_{K}=\alpha_{K} e^{1 /\left(2 s_{K}^{2} N_{K}\right)}$ and $s_{K}^{2} N_{K}$ is given in Eq. (C7). This predicts, for large $N_{T}$ and $K$, the
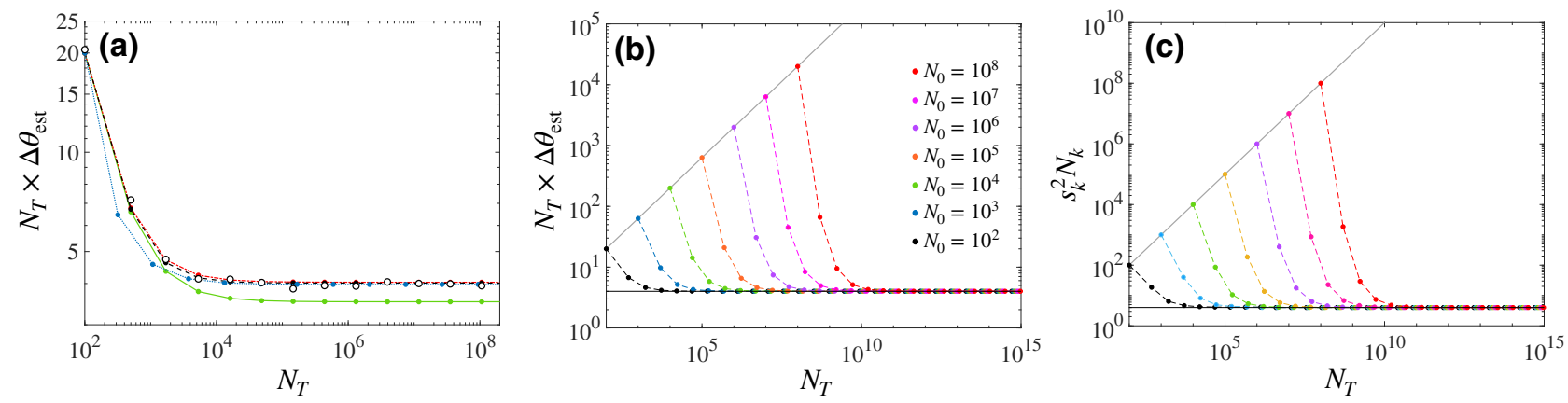

FIG. 7. (a) Sensitivity $\Delta^{2} \theta_{\text {est }}$ as a function of the total number of particles $N_{T}$ for a given initial $N_{0}\left(\right.$ here $\left.N_{0}=100\right)$. The green dots are calculated from Eq. (12), reaching Eq. (C8) asymptotically in $N_{T}$. The red dots are calculated from Eq. (C11), reaching Eq. (C12) asymptotically in $N_{T}$. The black dots are obtained from the numerical optimization of $s_{k}$ at each step, while using the analytical optimal sequence $N_{1}=4 N_{0}$ and $N_{k+1}=3 N_{k}$ for $k \geq 1$. The blue dots are obtained from the numerical optimization of $s_{k}$ and $N_{k}$ at each step. Lines are guides to the eye. (b) Sensitivity as a function of the total number of particles $N_{T}$ for different values of $N_{0}$ (different colors). Points are obtained from a full optimization over $s_{k}$ and $N_{k}$ at each step $\left(k=1, \ldots, K\right.$ and $\left.s_{0}=1\right)$; dashed lines are guides to the eye. The gray line is $\Delta \theta=2 / \sqrt{N_{T}}$. The horizontal black line is Eq. (C14). (c) Quantity $s_{k}^{2} N_{k}$ as a function of $N_{T}$ for different values of $N_{0}$ [same as in panel (b)]. The gray line is the case $s_{0}=1$, while the horizontal black line is Eq. (C15). 
sensitivity

$$
\Delta \theta_{\mathrm{est}}=\frac{4.034}{N_{T}},
$$

where the prefactor is $\alpha_{\infty} e^{1 / \sqrt{2 \times 3^{3}}}$. This value is closer than Eq. (C6) to the optimal numerical result (see below).

(b) We can also improve the prediction of Eq. (C6) by keeping the sequence of $N_{k}$ particles as discussed above, namely $N_{1}=4 N_{0}$ and $N_{k+1}=3 N_{k}$ for $1 \leq k \leq K-1$, and choose the optimal squeezing parameter $s_{k}$ at step $k$ by numerically solving the Eq. (C2), recursively, with $E\left[\left(\Delta \Theta_{0}\right)^{2}\right]=4 / N_{0}$ as initial condition. In this case, we obtain a sensitivity

$$
\Delta \theta_{\mathrm{est}}=\frac{4.01}{N_{T}} .
$$

(c) Finally, we have performed a full numerical optimization over $N_{k}$ and $s_{k}$ (for $k \geq 1$ ). For large $N_{0}$, the optimal sequence is $N_{1} \approx 2.2 N_{0}$ and $N_{k+1}=$ $3.5 N_{k}$ for $k \geq 1$, which is only slightly different from the optimal sequence found analytically. The full numerical optimization provides

$$
\Delta \theta_{\mathrm{est}}=\frac{3.97}{N_{T}}
$$

independently from the initial $N_{0}$; see Fig. 7(b). The asymptotic value of $s_{k}^{2} N_{k}$ for $k \rightarrow \infty$ is

$$
s_{\infty}^{2} N_{\infty}=4.009
$$

[see Fig. 7(c)], which is slightly larger than the analytical prediction (C8).

In conclusion, the analytical study and the numerical analysis fully support our claim that the phase estimation protocol reaches a sensitivity at the Heisenberg scaling $4 / N_{T}$, as discussed in the main text.

\section{APPENDIX D: IMPACT OF NOISE AND OTHER POSSIBLE IMPERFECTIONS}

\section{Number of particle fluctuations}

When implementing our quantum phase estimation protocol with atomic spin-squeezed states, it important to consider the impact of fluctuations of the atom number. We take into account this effect by replacing state (A1) with the statistical mixture

$$
\begin{aligned}
\hat{\rho}\left(\bar{N}, s, \sigma_{N}\right)= & \sum_{N=0}^{+\infty} \frac{e^{-\frac{(N-\bar{N})^{2}}{2 \sigma_{N}^{2}}}}{n} \\
& \sum_{\mu, \nu=-N / 2}^{N / 2} e^{-\left(\mu^{2}+v^{2}\right) /\left(s^{2} \bar{N}\right)}|N, \mu\rangle_{y}\langle N, \nu|,
\end{aligned}
$$

where $n$ provides the normalization $(\operatorname{Tr}[\hat{\rho}]=1), \bar{N}=$ $\operatorname{Tr}[\hat{\rho} \hat{N}]$ is the average number of particles, and the $|N, \mu\rangle_{y}$ are common eigenstates of $\hat{J}_{y}$ with eigenvalue $\mu$ and of the Casimir invariant of $\hat{J}_{z}^{2}+\hat{J}_{y}^{2}+\hat{J}_{z}^{2}$ with eigenvalue $(N / 2)(N / 2+1)$. This state reduces to Eq. (A1) in the limit $\sigma_{N} \rightarrow 0$. The calculation of the mean value and variances of average spin moments gives

$$
\begin{aligned}
\left\langle\hat{J}_{x}\right\rangle_{\hat{\rho}} & =\frac{\bar{N}}{2} e^{+1 /\left(2 s^{2} \bar{N}\right)} \\
\left(\Delta \hat{J}_{x}\right)_{\hat{\rho}}^{2} & =\frac{\sigma_{N}^{2}+\bar{N}^{2}}{8}\left(1+e^{-2 /\left(s^{2} \bar{N}\right)}\right)^{2}-\frac{\bar{N}^{2}}{4} e^{-1 /\left(s^{2} \bar{N}\right)} \\
& \approx \frac{\bar{N}^{2}}{8}\left(1-e^{-1 /\left(s^{2} \bar{N}\right)}\right)^{2}
\end{aligned}
$$

for $s^{2} \bar{N} \gtrsim 1$ and $\sigma_{N}^{2} \ll \bar{N}^{2}$ (note that, typically, we can consider $\sigma_{N}^{2}=\bar{N}$ and the condition $\sigma_{N}^{2} \ll \bar{N}^{2}$ is fulfilled

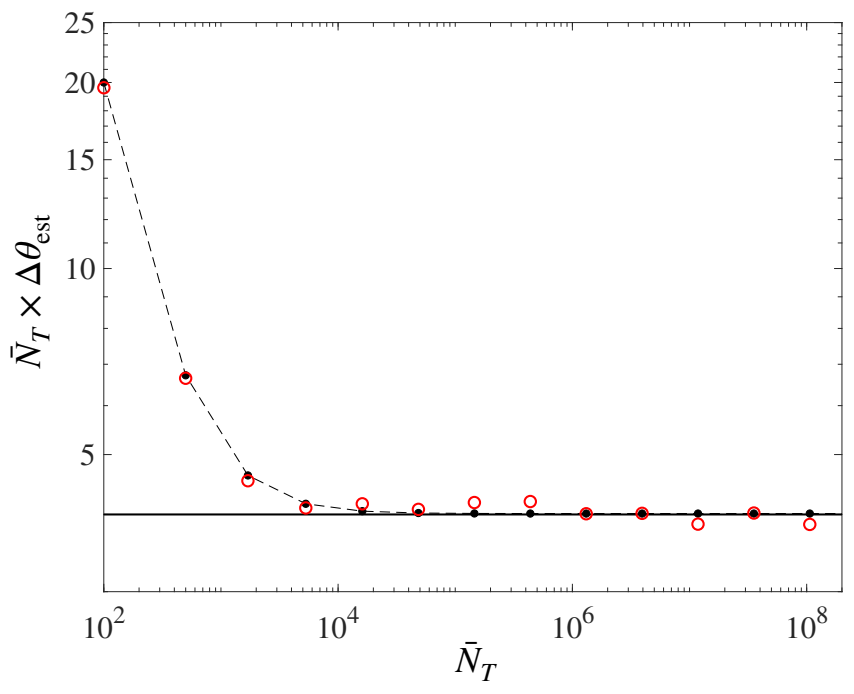

FIG. 8. Phase estimation with states of a fluctuating number of particles, Eq. (D1). The figure shows the phase uncertainty $\Delta \theta_{\text {est }}$ as a function of the total average number of particles $\bar{N}_{T}$. Red circles are results of numerical Monte Carlo simulations taking $\theta$ as a random phase in $[-\pi, \pi]$. Dots are expected semianalytical predictions with the dashed line being a guide to the eye. The solid black line is $4 / \bar{N}_{T}$ and the protocol starts with $\bar{N}_{0}=100$ particles. 
for $\bar{N} \gg 1$ ), and

$$
\left(\Delta \hat{J}_{y}\right)_{\hat{p}}^{2}=\frac{s^{2} \bar{N}}{4} .
$$

These equations are analogous to Eqs. (A3), (A9) and (A10), respectively, with the replacement $N \rightarrow \bar{N}$. The protocol discussed above, in the case of a fixed $N$, can thus be straightforwardly generalized to this case, providing the sensitivity $4 / \bar{N}_{T}$ with $\bar{N}_{T}=\sum_{k=1}^{K} \bar{N}_{k}$ being the total average number of particles.

In Fig. 8 we show results of Monte Carlo simulations of the protocol where the total number of particles is chosen randomly at each step $k=0,1, \ldots, K$ within a Gaussian distribution of variance $\sigma_{N_{k}}^{2}=\bar{N}_{k}$ and the average number of particles is taken in the sequence $\bar{N}_{1}=4 \bar{N}_{0}$ and $\bar{N}_{k+1}=$ $3 \bar{N}_{k}$ for $1 \leq k \leq K-1$.

\section{Collective dephasing}

We now study the effect of dephasing in the state preparation, while neglecting noise during the phase shift operation, assuming that these take a negligible time with respect to the decoherence time scale. Upon an additional rotation of the probe states $|\psi(N, s)\rangle$, we take the $y$ axis as the dephasing axis. We describe collective dephasing by the transformation $\hat{\rho}(N, s)=\Lambda_{y}[|\psi(N, s)\rangle]$, giving the density matrix

$$
\begin{aligned}
\hat{\rho}(N, s)= & \int_{-\pi}^{\pi} d \phi P(\phi) e^{-i \phi \hat{J}_{y}} \\
& \times|\psi(N, s)\rangle\langle\psi(N, s)| e^{i \phi \hat{J}_{y}}
\end{aligned}
$$

where the states $|\psi(N, s)\rangle$ are given in Eq. (A1), and $e^{-i \phi \hat{J}_{y}}$ has the physical meaning of a stochastic phase rotation around the $y$ axis with a probability distribution $P(\phi)$ [with $\left.\int_{-\pi}^{\pi} d \phi P(\phi)=1\right]$. We have

$$
\begin{gathered}
\left\langle\hat{J}_{x}\right\rangle_{\hat{\rho}}=\left\langle\hat{J}_{x}\right\rangle_{|\psi\rangle} e_{1}, \\
\left(\Delta \hat{J}_{x}\right)_{\hat{\rho}}^{2}=\left\langle\hat{J}_{x}^{2}\right\rangle_{|\psi\rangle} C_{2}-\left\langle\hat{J}_{x}\right\rangle_{|\psi\rangle}^{2} e_{1}^{2}+\left\langle\hat{J}_{z}^{2}\right\rangle_{|\psi\rangle} S_{2}, \\
\left(\Delta \hat{J}_{y}\right)_{\hat{\rho}}^{2}=\left(\Delta \hat{J}_{y}\right)_{|\psi\rangle}^{2}=s^{2} N / 4,
\end{gathered}
$$

where

$$
\begin{aligned}
& \mathcal{C}_{1}=\int_{-\pi}^{\pi} d \phi P(\phi) \cos \phi, \quad e_{2}=\int_{-\pi}^{\pi} d \phi P(\phi) \cos ^{2} \phi, \\
& \delta_{2}=\int_{-\pi}^{\pi} d \phi P(\phi) \sin ^{2} \phi .
\end{aligned}
$$

Here the pendix $|\psi\rangle$ refers to the mean values and variances of collective spin operators calculates for the state $|\psi(N, s)\rangle$. The $y$ depolarization leaves unchanged the moments of $\hat{J}_{y}$-in particular, it does not change the squeezing along the $y$ axis - but it decreases the length of the collective spin, $\left\langle\hat{J}_{x}\right\rangle$, and increases the bending of the state in the sphere, namely $\left\langle\hat{J}_{x}^{2}\right\rangle$. Substituting values (D5)-(D7) into Eq. (6) and following the protocol discussed above, we obtain the recursive relation for step $k$ [we assume that the same dephasing noise $P(\phi)$ affects all states in the phase estimation protocol],

$$
\mathcal{E}\left[\left(\Delta \Theta_{k}\right)^{2}\right]=\frac{2 s_{k}^{2}+N_{k}\left[1+\left(C_{2}-\delta_{2}\right) e^{-2 /\left(s_{k}^{2} N_{k}\right)}-2 C_{1}^{2} e^{-1 /\left(s_{k}^{2} N_{k}\right)}\right] \mathcal{E}\left[\left(\Delta \Theta_{k-1}\right)^{2}\right]}{2 N_{k} e^{-1 /\left(s_{k}^{2} N_{k}\right)} C_{1}^{2}}
$$

which assumes that $\mathscr{E}\left[\left(\Delta \Theta_{k-1}\right)^{2}\right] \ll 1$ in order to neglect higher-order expansion terms in the tangent function, and recovers Eq. (C1) in the case $C_{1,2}=1$ and $\delta_{2}=0$. The optimization over $s_{k}$ leads to the equation

$$
\begin{aligned}
& s_{k}^{4} N_{k}^{2}-s_{k}^{2} N_{k}+N_{k}^{2}\left(\frac{\left(C_{2}-\delta_{2}\right) e^{-2 /\left(s_{k}^{2} N_{k}\right)}-1}{2}\right) \\
& \times \mathscr{E}\left[\left(\Delta \Theta_{k-1}\right)^{2}\right]=0 .
\end{aligned}
$$

Figure 9 shows the results of a numerical optimization over both $s_{k}$ and $N_{k}$, and for different values of $N_{0}$. The noise distribution is conveniently chosen as

$$
P(\phi)=\frac{e^{\gamma \cos \phi}}{2 \pi I_{0}(\gamma)},
$$

where $I_{0}(x)$ is the modified Bessel function and $\gamma$ is the dephasing rate. In the case $\gamma \gg 1, P(\phi)$ can be well approximated as a Gaussian with width $\sigma_{\phi}^{2}=1 / \gamma$. Overall, we see that the protocol is very robust to $z$ depolarization noise: for large $K$, it reaches a Heisenberg scaling

$$
\Delta \theta_{\mathrm{est}}=\frac{\alpha_{K}^{\mathrm{deph}}}{N_{T}},
$$




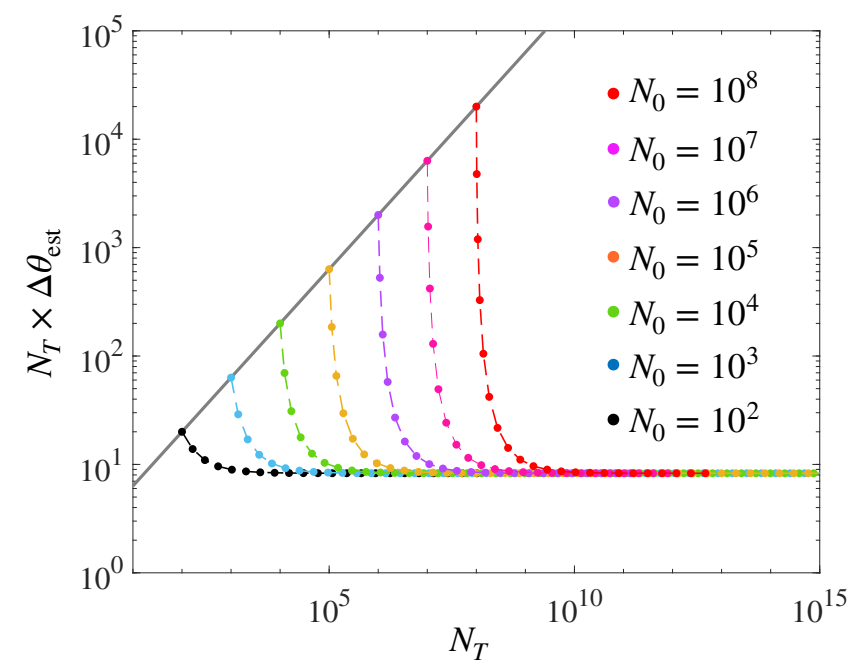

FIG. 9. Phase estimation in the presence of collective dephasing. Phase sensitivity $\Delta \theta_{\text {est }}$ as a function of $N_{T}$, for $y$-dephasing noise parameter $\gamma=4$ [see Eq. (D10)] and for different values of $N_{0}$. Circles are results of the numerical optimization of the recursive relation (D8) over $s_{k}$ and $N_{k}$ for $k=1, \ldots, K$. Dashed lines are guides to the eye. The solid line is $\Delta \theta=2 / \sqrt{N_{T}}$.

independently of $N_{0}$, where the prefactor $\alpha_{K}^{\text {deph }}$ depends on $e_{1}, C_{2}$, and $\delta_{2}$. In Fig. 9 we show the expected phase sensitivity as a function of $N_{T}$ for $\gamma=4$ and different values of $N_{0}$. Symbols are obtained by numerically optimizing Eq. (D8) over $N_{k}$ and $s_{k}$ at each step. Furthermore, in Fig. 3(a) of the main text we show a comparison between the numerical optimization and Monte Carlo simulations of the protocol. In Fig. 3(b) of the main text we plot the prefactor $\alpha_{K}^{\text {deph }}$ as a function of $\gamma$.

\section{Full depolarization}

We consider full depolarization within the symmetric subspace of dimension $N+1, \hat{\rho}(N, s)=\Lambda_{\mathscr{D}}[|\psi(N, s)\rangle]$, with

$$
\begin{aligned}
\hat{\rho}(N, s)= & (1-\mathscr{D})|\psi(N, s)\rangle\langle\psi(N, s)| \\
& +\frac{\mathscr{D}}{N+1} \mathbb{1},
\end{aligned}
$$

where $0 \leq \mathscr{D} \leq 1$. The mean value of any collective operator $\hat{J}_{n}^{\alpha}$ becomes

$$
\left\langle\hat{J}_{n}^{\alpha}\right\rangle_{\hat{\rho}}=(1-\mathscr{D})\left\langle\hat{J}_{n}^{\alpha}\right\rangle_{|\psi\rangle}+\frac{\mathscr{D}}{N+1} \operatorname{Tr}\left[\hat{J}_{n}^{\alpha}\right],
$$

for any direction $n$ and any power $\alpha$. Taking into account the facts that

$$
\begin{aligned}
\operatorname{Tr}\left[\hat{J}_{n}\right] & =0 \quad \text { and } \operatorname{Tr}\left[\hat{J}_{n}^{2}\right]=\sum_{\mu=-N / 2}^{N / 2} \mu^{2} \\
& =\frac{N(N+1)(N+2)}{12}
\end{aligned}
$$

and using Eqs. (A3) and (A9) in Eq. (A10), for $s^{2} N \gtrsim 1$ we have

$$
\left\langle\hat{J}_{x}\right\rangle_{\hat{\rho}}=\frac{(1-\mathscr{D}) N}{2} e^{-1 /\left(2 s^{2} N\right)},
$$

$$
\begin{aligned}
\left(\Delta \hat{J}_{x}\right)_{\hat{\rho}}^{2}= & \frac{(1-\mathscr{D}) N^{2}}{8}\left[1+e^{-2 /\left(s^{2} N\right)}-2(1-\mathscr{D}) e^{-1 /\left(s^{2} N\right)}\right] \\
& +\frac{\mathscr{D} N(N+2)}{12}, \\
\left(\Delta \hat{J}_{y}\right)_{\hat{\rho}}^{2}=\frac{(1-\mathscr{D}) s^{2} N}{4}+\frac{\mathscr{D} N(N+2)}{12} . & \text { (D16) }
\end{aligned}
$$

It should be noted that, according to Eq. (D17), the variance $\left(\Delta \hat{J}_{y}\right)_{\hat{\rho}}^{2}$ cannot be smaller than $\mathscr{D} N(N+2) / 12$. Identifying this value as $s_{\min }^{2} N / 4$, we obtain a smallest achievable squeezing parameter $s_{\min }^{2} \gtrsim \mathscr{D} N / 3$, meaning that, when a state (A1) with squeezing parameter $s \ll$ $s_{\min }$ is depolarized according to Eq. (D12), it does not lead to a decrease of variance $\left(\Delta \hat{J}_{y}\right)_{\hat{\rho}}^{2}$ below $\mathscr{D} N(N+$ 2)/12. Substituting the above spin moments into Eq. (6), we obtain the recursive relation for the $K$ th step of the algorithm

$$
\begin{aligned}
\mathscr{E}\left[\left(\Delta \Theta_{k}\right)^{2}\right]= & \frac{3(1-\mathscr{D}) s_{k}^{2}+\mathscr{D}\left(N_{k}+2\right)}{3(1-\mathscr{D})^{2} N_{k} e^{-1 /\left(s_{k}^{2} N_{k}\right)}} \\
& +\frac{3(1-\mathscr{D}) N_{k}\left[1+e^{-2 /\left(s_{k}^{2} N_{k}\right)}-2(1-\mathscr{D}) e^{-1 /\left(s_{k}^{2} N_{k}\right)}\right]+2 \mathscr{D}\left(N_{k}+2\right)}{6(1-\mathscr{D})^{2} N_{k} e^{-1 /\left(s_{k}^{2} N_{k}\right)}} \mathscr{E}\left[\left(\Delta \Theta_{k-1}\right)^{2}\right] .
\end{aligned}
$$

In the following we restrict our study to the case $\mathscr{D} \ll 1$. In Fig. 11 we plot the numerical optimization of recursive relation (D18) for $\mathscr{D}=10^{-8}$, taking $N_{0}=100$ and $s_{0}=1$ as initial conditions. The value $s_{\min }^{2}=\mathscr{D} N / 3$ identifies a 


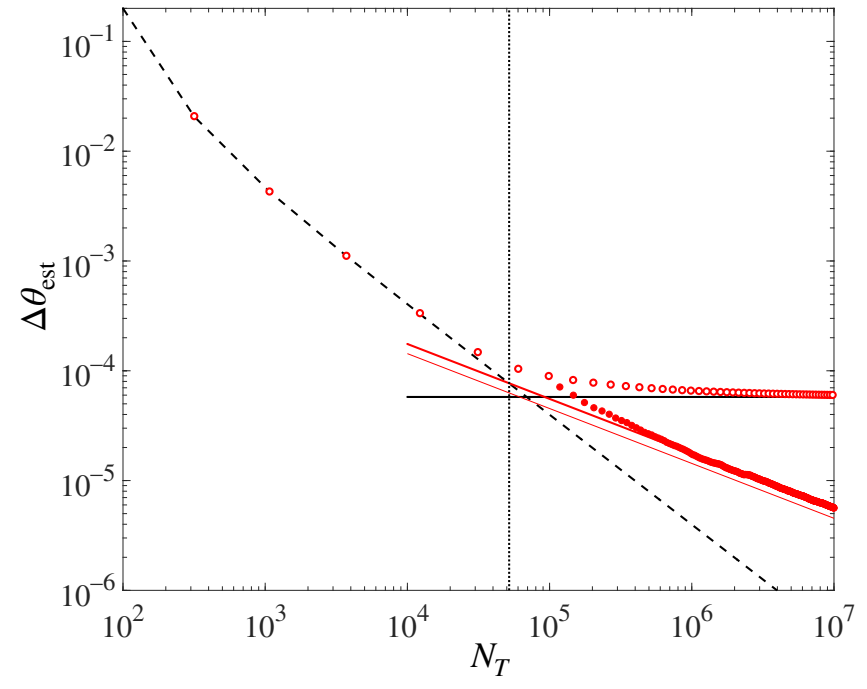

FIG. 10. Phase estimation in the presence of full depolarization. Phase sensitivity $\Delta \theta_{\text {est }}$ as a function of $N_{T}$ for full depolarization noise with parameter $\mathscr{D}=10^{-8}$ and $N_{0}=100$. Open circles are the results of the numerical optimization over $s_{k}$ and $N_{k}$ of recursive relation (D18). The black dashed line is the ideal noiseless case $(\mathscr{D}=0)$ that follows the Heisenberg scaling $(\mathrm{C} 14)$ for large $N_{T}$. The black horizontal line is Eq. (D19), which predicts a saturation of $\Delta \theta_{\text {est }}$ at a value fully identified by $\mathscr{D}$. The vertical dotted line corresponds to the critical $\tilde{N}_{T}$, Eq. (D20), which identifies the transition from a noiseless regime to a noisedominated one. The dots are obtained by repeating the estimation protocol with a depolarized squeezed state of $N_{\tilde{k}}$ particles and squeezing parameter $\tilde{k}$. The thin solid red line is Eq. (D21), while the thick solid red line is a fit $\Delta \theta_{\text {est }}=\beta_{D} / \sqrt{N_{T}}$ for large $N_{T}$.

critical step $\tilde{k}\left(s_{\min }=s_{\tilde{k}}\right)$. For $k \lesssim \tilde{k}$, the protocol essentially follows the ideal protocol and depolarization noise does not play any major role. For $k \gtrsim \tilde{k}$, the sensitivity quickly saturates to the value

$$
\Delta^{2} \theta_{\text {est }, k \gtrsim \tilde{k}}=\frac{\mathscr{D}}{3(1-\mathscr{D})^{2}} .
$$

The critical value $\tilde{k}$ can be obtained by using the relation $s_{\tilde{k}}^{2} N_{\tilde{k}}=4$ [see Eq. (C14)], assuming that $\tilde{k} \gg 1$ (which is valid for $\mathscr{D} \ll 1$ ). Using $s_{\tilde{k}}^{2} s_{2}=\mathscr{D} N_{\tilde{k}} / 3$, we obtain $N_{\tilde{k}}^{2}=12 / \mathscr{D}$ and, using Eq. (10), we have $\tilde{k}=$ $\log _{3}\left[3 \sqrt{3} /\left(2 N_{0} \sqrt{\mathcal{E}}\right)\right]$. This value corresponds to a total critical number of particles

$$
\tilde{N}_{T}=\sum_{k=0}^{\tilde{k}} N_{k}=3 \sqrt{\frac{3}{\mathscr{D}}},
$$

which is obtained by noting that $\tilde{N}_{T}=3 N_{\tilde{k}} / 2$ and is highlighted by the vertical dotted line in Fig. 11 . The corresponding sensitivity reached by the protocol at this point is approximatively $\Delta \theta_{\text {est }, \tilde{k}} \approx 4 / \tilde{N}_{T}$, according to Eq. (C13). For $k \gtrsim \tilde{k}$, it is convenient to modify the algorithm by avoiding phase feedback between the different steps of the protocol and repeating the estimation using multiple copies of $\left|\psi\left(N_{\tilde{k}}, s_{\tilde{k}}\right)\right\rangle$ [which are depolarized according to Eq. (D12)]. Using $m$ copies of the state gives a statistical gain factor $1 / \sqrt{m}$ in sensitivity, such that

$$
\Delta \theta_{\mathrm{est}} \approx \frac{4}{\tilde{N}_{T} \sqrt{m}}=\frac{8}{3}\left(\frac{\mathscr{D}}{12}\right)^{1 / 4} \frac{1}{\sqrt{N_{T}}} .
$$

To derive this equation we have used $N_{T}=\tilde{N}_{T}+m N_{\tilde{k}} \approx$ $m N_{\tilde{k}}$ for $m \gg 1$ since $\tilde{N}_{T}=3 N_{\tilde{k}} / 2$. Equation (D21), shown by the thin solid red line in Fig. 11, slightly underestimates the prefactor $\beta_{\mathscr{D}}$ of the asymptotic $\beta_{\mathscr{D}} / \sqrt{N_{T}}$ scaling of sensitivity. Nevertheless, Eq. (D21) accurately catches the behavior $\beta_{\mathscr{D}} \propto \mathscr{D}^{1 / 4}$, as shown in Fig. 3(d) of the main text.

\section{Particle losses}

To show the robustness of our QPE protocol against losses, we consider a simple model. We focus on bosonic modes, $a$ and $b$, and model the loss of particles by coupling to additional modes, $\hat{A}$ and $\hat{B}$, respectively, that are initially in the vacuum state: $\hat{a}_{\eta}=\sqrt{1-\eta} \hat{a}+\sqrt{\eta} \hat{A}$ and

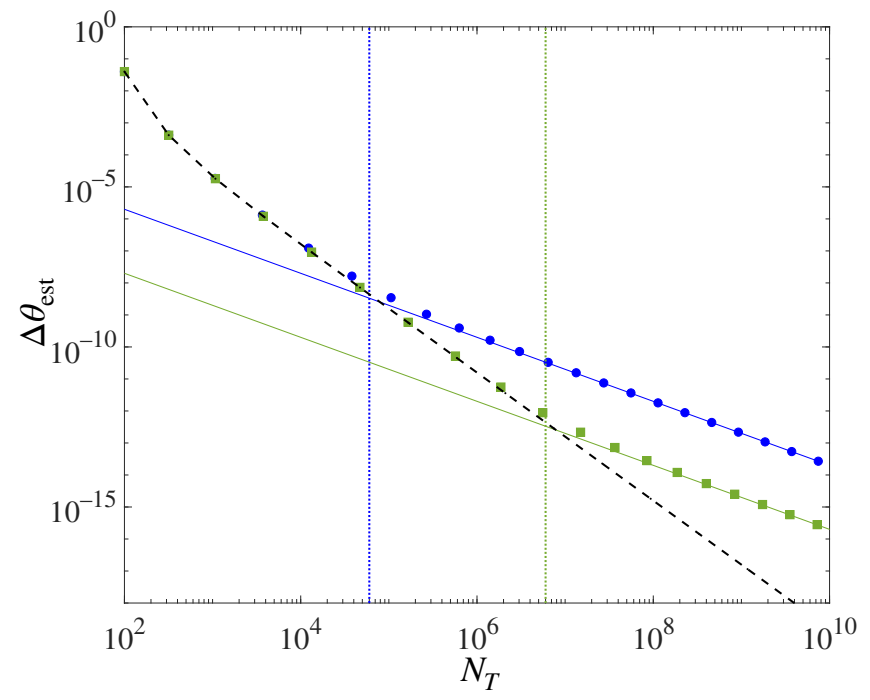

FIG. 11. Phase estimation in the presence of losses. Phase sensitivity $\Delta \theta_{\text {est }}$ as a function of $N_{T}$ in the presence of losses. Symbols are obtained by optimizing recursive relation (D25) over $s_{K}$ and $N_{K}$ for $k=1 ; \ldots, K$ : blue dots are obtained for $\eta=10^{-4}$ and green squares for $\eta=10^{-6}$. The respective solid lines are calculated from Eq. (D26). The black dashed line is the ideal noiseless case $(\eta=0)$ that follows the Heisenberg scaling (C13) for large $N_{T}$. Vertical dotted lines are $\tilde{N}_{T}=6 /\left(\eta-\eta^{2}\right)$ (see the text). The initial number of particles is $N_{0}=100$. 
$\hat{b}_{\eta}=\sqrt{1-\eta} \hat{b}+\sqrt{\eta} \hat{B}$, where $\eta$ is a loss coefficient ranging from 0 (no loss) to 1 (full particle loss from $a$ and $b$ modes). Considering $\hat{J}_{x}=\left(\hat{a}_{\eta}^{\dagger} \hat{b}_{\eta}+\hat{b}_{\eta}^{\dagger} \hat{a}_{\eta}\right) / 2, \hat{J}_{y}=\left(\hat{a}_{\eta}^{\dagger} \hat{b}_{\eta}-\right.$ $\left.\hat{b}_{\eta}^{\dagger} \hat{a}_{\eta}\right) / 2 i, \hat{J}_{z}=\left(\hat{a}_{\eta}^{\dagger} \hat{a}_{\eta}-\hat{b}_{\eta}^{\dagger} \hat{b}_{\eta}\right) / 2$, we have

$$
\begin{gathered}
\left\langle\hat{J}_{x}\right\rangle_{\eta}=\frac{(1-\eta) N}{2} e^{-1 /\left(2 s^{2} N\right)}, \\
\left(\Delta \hat{J}_{x}\right)_{\eta}^{2}=\frac{(1-\eta)^{2} N^{2}}{8}\left(1-e^{-1 /\left(s^{2} N\right)}\right)^{2}+\frac{\eta(1-\eta) N}{4} \\
\left(\Delta \hat{J}_{y}\right)_{\eta}^{2}=\frac{(1-\eta)^{2} s^{2} N}{4}+\frac{\eta(1-\eta) N}{4} .
\end{gathered}
$$

Similar to the case of full depolarization, there is a lower bound to $\left(\Delta \hat{J}_{y}\right)_{\eta}^{2}$ : it cannot be smaller than $N \eta(1-\eta) / 4$. From the relation $N s_{\min }^{2} / 4=N \eta(1-\eta) / 4$ we identify a smallest squeezing parameter $s_{\min }^{2}=$ $\eta(1-\eta)$. For $\eta \ll 1$, we can thus identify critical algorithm step $\tilde{k}$, such that, for $k \lesssim \tilde{k}$, the noisy algorithm follows the ideal noiseless one. Using $s_{\tilde{k}}^{2} N_{\tilde{k}}=$ $4, s_{k}=s_{\min }$ and $\tilde{N}_{T}=3 N_{\tilde{k}} / 2$, we obtain $\tilde{N}_{T}=6 /$ $\left(\eta-\eta^{2}\right)$.

We substitute the above spin moments into Eq. (6) and obtain the recursive relation for the $k$ th step of the algorithm

$$
\mathcal{E}\left[\left(\Delta \Theta_{k}\right)^{2}\right]=\frac{2(1-\eta) s_{k}^{2}+2 \eta+\left[(1-\eta) N_{k}\left(1-e^{-1 /\left(s_{k}^{2} N_{k}\right)}\right)^{2}+2 \eta\right] \mathscr{E}\left[\left(\Delta \Theta_{k-1}\right)^{2}\right]}{2(1-\eta) N_{k} e^{-1 /\left(s^{2} N_{k}\right)}} .
$$

For $N_{T} \lesssim \tilde{N}_{T}$, we thus achieve the Heisenberg scaling $\Delta \theta_{\text {est }}=4 / N_{T}$. For $N_{T} \gtrsim \tilde{N}_{T}$, the noise associated with particle losses dominates the algorithm. It is more convenient to repeat the phase estimation using $m$ copies of the state $\left|\psi\left(N_{\tilde{k}}, s_{\tilde{k}}\right)\right\rangle$. The sensitivity thus follows

$$
\Delta \theta_{\mathrm{est}}=\sqrt{\frac{\eta}{(1-\eta)}} \frac{1}{\sqrt{N_{T}}} .
$$

\section{APPENDIX E: NONLINEAR READOUT}

As discussed in the main text, the output state of the $k$ th step of the protocol is now

$\left|\phi_{k}^{\text {out }}\left(N_{k}, \tau_{k}, \theta_{k}\right)\right\rangle=e^{-i(\pi / 2) \hat{J}_{x}} e^{+i \tau_{k} \hat{J}_{z}^{2}} e^{-i \theta_{k} \hat{J}_{y}} e^{-i \tau_{k} \hat{J}_{z}^{2}}\left|\operatorname{CSS}\left(N_{k}\right)\right\rangle$.

$$
\mathcal{E}\left[\left(\Delta \Theta_{k}\right)^{2}\right]=\int_{-\ell_{k}}^{\ell_{k}} d \theta_{k}\left(\frac{\left(\Delta \hat{J}_{z}^{\text {out }}\right)_{k}^{2}+\sigma_{\mathrm{DN}}^{2}}{\left(d\left\langle\hat{J}_{z}^{\text {out }}\right\rangle_{k} / d \theta_{k}\right)^{2}}\right) P\left(\theta_{k}\right),
$$
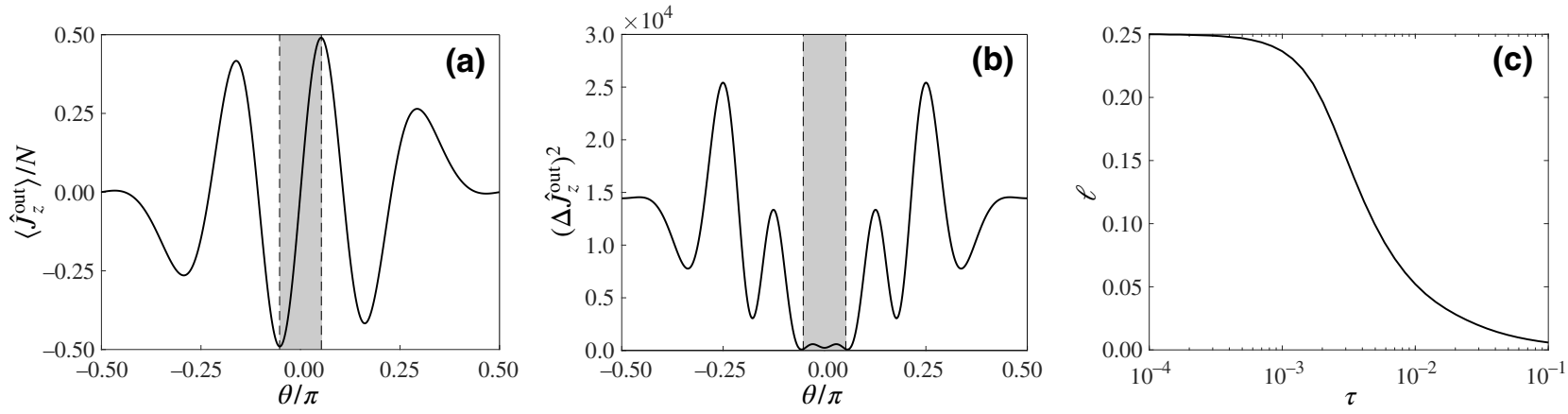

FIG. 12. Plots of (a) $\left\langle\hat{J}_{z}^{\text {out }}\right\rangle$ and (b) $\left(\Delta \hat{J}_{z}^{\text {out }}\right)^{2}$ as a function of $\theta$ for $N=1000$ and $\tau=0.01$. The shaded region indicates the inversion region $\theta \in[-\ell, \ell]$ around $\theta=0$ where $\left\langle\hat{J}_{z}^{\text {out }}\right\rangle$ is monotonic. Panel (c) shows $\ell$ as a function of $\tau$. 
TABLE I. Optimization parameters $N_{k}$ and $\tau_{k}$ used in Fig. 4 of the main text in the case $\sigma_{\mathrm{DN}}^{2}=0$.

\begin{tabular}{lcc}
\hline \hline Step & $N$ & $\tau$ \\
\hline 0 & 600 & 0 \\
1 & 500 & $6.6 \times 10^{-3}$ \\
2 & 2400 & $5.8 \times 10^{-3}$ \\
3 & 6600 & $5.5 \times 10^{-3}$ \\
\hline \hline
\end{tabular}

where $\sigma_{\mathrm{DN}}^{2}$ is the detection noise parameter, and $P\left(\theta_{k}\right)$ is taken as a Gaussian distribution centered at $\theta_{k}=0$ and of variance $\mathcal{E}\left[\left(\Delta \Theta_{k-1}\right)^{2}\right]$. The optimal values of $\tau_{k}$ and $N_{k}$ are found by minimizing $\mathcal{E}\left[\left(\Delta \Theta_{k}\right)^{2}\right] \times \sum_{j=0}^{k} N_{j}$, with initial conditions $\mathscr{E}\left[\left(\Delta \Theta_{0}\right)^{2}\right]=4 / N_{0}$. In particular, we find that, for the optimal parameters, the quantity $2 \int_{0}^{\ell_{k}} d \theta_{k} P\left(\theta_{k}\right)$ is negligible. In Table I we list the values of $\tau_{k}$ and $N_{k}$ used in the numerical simulations giving Fig. 4 of the main text in the case $\sigma_{\mathrm{DN}}^{2}=0$. For $\sigma_{\mathrm{DN}}^{2} \neq 0$, we have optimized only with respect to $\tau_{k}$, obtaining values similar to the noiseless case.

[1] A. Yu. Kitaev, Quantum measurements and the Abelian stabilizer problem, Electron. Colloq. Comput. Complex. 3 (1996), ArXiv:quant-ph/9511026.

[2] A. Yu. Kitaev, A. Shen, and M. Vyalyi, Classical and Quantum Computation (American Mathematical Society, Providence, Rhode Island, 2002).

[3] R. Cleve, A. Ekert, C. Macchiavello, and M. Mosca, Quantum algorithms revisited, Proc. R. Soc. A 454, 339 (1998).

[4] M. A. Nielsen and I. L. Chuang, Quantum Computation and Quantum Information (Cambridge University Press, Cambridge, 2001).

[5] D. S. Abrams and S. Lloyd, Quantum Algorithm Providing Exponential Speed Increase for Finding Eigenvalues and Eigenvectors, Phys. Rev. Lett. 83, 5162 (1999).

[6] A. Aspuru-Guzik, A. D. Dutoi, P. J. Love, and M. HeadGordon, Simulated quantum computation of molecular energies, Science 309, 1704 (2005).

[7] S. McArdle, S. Endo, A. Aspuru-Guzik, S. C. Benjamin, and X. Yuan, Quantum computational chemistry, Rev. Mod. Phys. 92, 015003 (2020).

[8] P. W. Shor, Polynomial-time algorithms for prime factorization and discrete logarithms on a quantum computer, SIAM J. Comput. 26, 1484 (1997).

[9] E. Martin-Lopez, A. Laing, T. Lawson, R. Alvarez, X.-Q. Zhou, and J. L. O'Brien, Experimental realization of Shor's quantum factoring algorithm using qubit recycling, Nat. Photonics 6, 773 (2012).

[10] T. Monz, D. Nigg, E. A. Martinez, M. F. Brandl, P. Schindler, R. Rines, S. X. Wang, I. L. Chuang, and R. Blatt, Realization of a scalable Shor algorithm, Science 351, 1068 (2016).

[11] K. Temme, T. J. Osborne, K. G. Vollbrecht, D. Poulin, and F. Verstraete, Quantum metropolis sampling, Nature 471, 87 (2011).
[12] T. Rudolph and L. Grover, Quantum Communication Complexity of Establishing a Shared Reference Frame, Phys. Rev. Lett. 91, 217905 (2003).

[13] M. de Burgh and S. D. Bartlett, Quantum methods for clock synchronization: Beating the standard quantum limit without entanglement, Phys. Rev. A 72, 042301 (2005).

[14] E. M. Kessler, P. Kómár, M. Bishof, L. Jiang, A. S. Sørensen, J. Ye, and M. D. Lukin, Heisenberg-Limited Atom Clocks Based on Entangled Qubits, Phys. Rev. Lett. 112, 190403 (2014).

[15] P. Kómár, E. M. Kessler, M. Bishof, L. Jiang, A. S. Sørensen, J. Ye, and M. D. Lukin, A quantum network of clocks, Nat. Phys. 10, 582 (2014).

[16] R. B. Griffiths and C.-S. Niu, Semiclassical Fourier Transform for Quantum Computation, Phys. Rev. Lett. 76, 3228 (1996).

[17] W. van Dam, M. D'Ariano, A. Ekert, C. Macchiavello, and M. Mosca, Optimal Quantum Circuits for General Phase Estimation, Phys. Rev. Lett. 98, 090501 (2007).

[18] B. L. Higgins, D. W. Berry, S. D. Bartlett, H. M. Wiseman, and G. J. Pryde, Entanglement-free Heisenberg-limited phase estimation, Nature 450, 393 (2007).

[19] S. Paesani, A. A. Gentile, R. Santagati, J. Wang, N. Wiebe, D. P. Tew, J. L. O'Brien, and M. G. Thompson, Experimental Bayesian Quantum Phase Estimation on a Silicon Photonic Chip, Phys. Rev. Lett. 118, 100503 (2017).

[20] C. Bonato, M. S. Blok, H. T. Dinani, D. W. Berry, M. L. Markham, D. J. Twitchen, and R. Hanson, Optimized quantum sensing with a single electron spin using real-time adaptive measurements, Nat. Nanotechnol. 11, 247 (2016).

[21] M. W. Mitchell, Metrology with entangled states, Proc. SPIE 5893, 589310 (2005).

[22] L. Pezzè and A. Smerzi, Sub shot-noise interferometric phase sensitivity with beryllium ions Schrödinger cat states, Europhys. Lett. 78, 30004 (2007).

[23] D. W. Berry, B. L. Higgins, S. D. Bartlett, M. W. Mitchell, G. J. Pryde, and H. M. Wiseman, How to perform the most accurate possible phase measurements, Phys. Rev. A 80, 052114 (2009).

[24] N. Wiebe and C. Granade, Efficient Bayesian Phase Estimation, Phys. Rev. Lett. 117, 010503 (2016).

[25] B. M. Escher, R. L. de Matos Filho, and L. Davidovich, General framework for estimating the ultimate precision limit in noisy quantum-enhanced metrology, Nat. Phys. 7, 406 (2011).

[26] R. Demkowicz-Dobrzański, J. Kolodyński, and M. Guta, The elusive Heisenberg limit in quantum-enhanced metrology, Nat. Commun. 3, 1063 (2012).

[27] T. Monz, P. Schindler, J. T. Barreiro, M. Chwalla, D. Nigg, W. A. Coish, M. Harlander, W. Hänsel, M. Hennrich, and R. Blatt, 14-Qubit Entanglement: Creation and Coherence, Phys. Rev. Lett. 106, 130506 (2011).

[28] A. Omran, H. Levine, A. Keesling, G. Semeghini, T. T. Wang, S. Ebadi, H. Bernien, A. S. Zibrov, H. Pichler, S. Choi, J. Cui, M. Rossignolo, P. Rembold, S. Montangero, T. Calarco, M. Endres, M. Greiner, V. Vuletic, and M. D. Lukin, Generation and manipulation of Schrödinger cat states in Rydberg atom arrays, Science 365, 570 (2019).

[29] A. S. Sørensen, L.-M. Duan, J. I. Cirac, and P. Zoller, Many-particle entanglement with Bose-Einstein condensates, Nature 409, 63 (2001). 
[30] L. Pezzè, A. Smerzi, M. K. Oberthaler, R. Schmied, and P. Treutlein, Quantum metrology with nonclassical states of atomic ensembles, Rev. Mod. Phys. 90, 035005 (2018).

[31] J. Ma, X. Wang, C. P. Sun, and F. Nori, Quantum spin squeezing, Phys. Rep. 509, 89 (2011).

[32] J. G. Bohnet, B. C. Sawyer, J. W. Britton, M. L. Wall, A. M. Rey, M. Foss-Feig, and J. J. Bollinger, Quantum spin dynamics and entanglement generation with hundreds of trapped ions, Science 352, 1297 (2016).

[33] C. Gross, T. Zibold, E. Nicklas, J. Estève, and M. K. Oberthaler, Nonlinear atom interferometer surpasses classical precision limit, Nature 464, 1165 (2010).

[34] M. F. Riedel, P. Böhi, Y. Li, T. W. Hänsch, A. Sinatra, and P. Treutlein, Atom-chip-based generation of entanglement for quantum metrology, Nature 464, 1170 (2010).

[35] J. Appel, P. J. Windpassinger, D. Oblak, U. B. Hoff, N. Kjærgaard, and E. S. Polzik, Mesoscopic atomic entanglement for precision measurements beyond the standard quantum limit, Proc. Natl. Acad. Sci. USA 106, 10960 (2009).

[36] I. D. Leroux, M. H. Schleier-Smith, and V. Vuletić, Implementation of Cavity Squeezing of a Collective Atomic Spin, Phys. Rev. Lett. 104, 073602 (2010).

[37] G. J. Bohnet, K. C. Cox, M. A. Norcia, J. M. Weiner, Z. Chen, and J. K. Thompson, Reduced spin measurement back-action for a phase sensitivity ten times beyond the standard quantum limit, Nat. Photonics 8, 731 (2014).

[38] O. Hosten, N. J. Engelsen, R. Krishnakumar, and M. A. Kasevich, Measurement noise 100 times lower than the quantum-projection limit using entangled atoms, Nature 529, 505 (2016).

[39] M.-Z. Huang, J. Alberto de la Paz, T. Mazzoni, K. Ott, A. Sinatra, C. L. Garrido Alzar, and J. Reichel, Selfamplifying spin measurement in a long-lived spin-squeezed state, ArXiv:2007.01964.

[40] H. Bao, J. Duan, S. Jin, X. Lu, P. Li, W. Qu, M. Wang, I. Novikova, E. E. Mikhailov, K.-F. Zhao, K. Mølmer, H. Shen, and Y. Xiao, Spin squeezing of $10^{11}$ atoms by prediction and retrodiction measurements, Nature 581, 159 (2020).

[41] D. J. Wineland, J. J. Bollinger, W. M. Itano, and D. J. Heinzen, Squeezed atomic states and projection noise in spectroscopy, Phys. Rev. A 50, 67 (1994).

[42] L. Pezzè and A. Smerzi, Entanglement, Nonlinear Dynamics, and the Heisenberg Limit, Phys. Rev. Lett. 102, 100401 (2009).

[43] Let us write the GSS Eq. (2) as $\left|\psi_{k}\right\rangle=\sum_{\mu=-N_{k} / 2}^{N_{k} / 2} c_{k}(\mu)|\mu\rangle_{z}$. Here, the $|\mu\rangle_{z}$ are eigenstates of $\hat{J}_{z}$ with eigenvalues $-N_{k} / 2 \leq \mu_{k} \leq N_{k} / 2$ and $c_{k}\left(\mu_{k}\right)=(1 / \sqrt{\eta}) \sum_{\nu=-N_{k} / 2}^{N_{k} / 2}$ $e_{z}^{-v^{2} /\left(s_{k}^{2} N_{k}\right)}\langle\mu \mid v\rangle_{y}$. Taking into account the facts that the $|\mu\rangle_{z}$ are given by the symmetrized combination on $N_{k} / 2+\mu$ particles in $|0\rangle$ and $N_{k} / 2-\mu$ in $|1\rangle$, and $|\mu\rangle_{z}=\operatorname{Sym}\left[|0\rangle^{\otimes\left(N_{k} / 2+\mu\right)} \otimes|1\rangle^{\otimes\left(N_{k} / 2-\mu\right)}\right] / \sqrt{\left(\begin{array}{l}N \\ \mu\end{array}\right)}$, we have $U_{c}^{\otimes N_{k}}\left|\psi_{k}\right\rangle|u\rangle=\sum_{\mu=-N_{k} / 2}^{N_{k} / 2} c_{k}(\mu) e^{i\left(N_{k} / 2-\mu\right) \theta}|\mu\rangle_{z}|u\rangle$ $=e^{-i \theta \hat{J}_{z}}\left|\psi_{k}\right\rangle|u\rangle$ a part a global (and irrelevant) phase factor $e^{i \theta_{k} N_{k} / 2}$.
[44] The assumption $s_{k}^{2} N_{k} \gg 1$ is only partially justified for large values of $k$. Higher order terms in Eq. (7) are explicitly calculated in Appendix B.

[45] Following Ref. [46], the Heisenberg limit is given by $\Delta \theta_{\mathrm{HL}}=1 /\left(H_{\max }-H_{\min }\right)$, where $H_{\max }$ and $H_{\min }$ are respectively the maximum and minimum eigenvalues of the collective Hamiltonian applied, via unitary phase encoding, to the overall probe state. In our case, the phase encoding Hamiltonian applied to $N_{T}$ qubits is identified as $\hat{H}=$ $\sum_{i=1}^{N_{T}} \hat{\sigma}_{z}^{(i)} / 2$, such that $H_{\max }=N_{T} / 2, H_{\min }=-N_{T} / 2$, and, thus, $\Delta \theta_{\mathrm{HL}}=1 / N_{T}$. The adaptive phase feedback can be identified as a specific measurement strategy included in the optimization leading to the abovementioned definition of the Heisenberg limit [47]. Recently, it has been shown that in the presence of an arbitrary but finite prior knowledge the standard definition of the Heisenberg limit should be corrected by a factor $\pi$ [48], giving $\Delta \theta_{\mathrm{HL}}=\pi / N_{T}$. We note that our adaptive method reaches $\Delta \theta_{\mathrm{HL}}=4 / N_{T}$, which is very close to the Heisenberg limit discussed in Ref. [48].

[46] V. Giovannetti, S. Lloyd, and L. Maccone, Quantum Metrology, Phys. Rev. Lett. 96, 010401 (2006).

[47] S. L. Braunstein and C. M. Caves, Statistical Distance and the Geometry of Quantum States, Phys. Rev. Lett. 72, 3439 (1994).

[48] W. Górecki, R. Demkowicz-Dobrzański, H. M. Wiseman, and D. W. Berry, $\pi$-Corrected Heisenberg Limit, Phys. Rev. Lett. 124, 030501 (2020).

[49] A. D. Ludlow, M. M. Boyd, J. Ye, E. Peik, and P. O. Schmidt, Optical atomic clocks, Rev. Mod. Phys. 87, 637 (2015).

[50] L. Pezzè and A. Smerzi, Heisenberg-Limited Noisy Atomic Clock Using a Hybrid Coherent and Squeezed States Protocol, Phys. Rev. Lett. 125, 210503 (2020).

[51] S. F. Huelga, C. Macchiavello, T. Pellizzari, A. K. Ekert, M. B. Plenio, and J. I. Cirac, Improvement of Frequency Standards with Quantum Entanglement, Phys. Rev. Lett. 79, 3865 (1997).

[52] E. Davis, G. Bentsen, and M. Schleier-Smith, Approaching the Heisenberg Limit without Single-Particle Detection, Phys. Rev. Lett. 116, 053601 (2016).

[53] F. Fröwis, P. Sekatski, and W. Dür, Detecting Large Quantum Fisher Information with Finite Measurement Precision, Phys. Rev. Lett. 116, 090801 (2016).

[54] T. Macrì, A. Smerzi, and L. Pezzè, Loschmidt echo for quantum metrology, Phys. Rev. A 94, 010102 (2016).

[55] O. Hosten, R. Krishnakumar, N. J. Engelsen, and M. A. Kasevich, Quantum phase magnification, Science 352, 1552 (2016).

[56] S. A. Haine, Using interaction-based readouts to approach the ultimate limit of detection-noise robustness for quantum-enhanced metrology in collective spin systems, Phys. Rev. A 98, 030303(R) (2018).

[57] M. Kitagawa and M. Ueda, Squeezed spin states, Phys. Rev. A 47, 5138 (1993).

[58] D. W. Berry and H. M. Wiseman, Optimal States and Almost Optimal Adaptive Measurements for Quantum Interferometry, Phys. Rev. Lett. 85, 5098 (2000).

[59] H. M. Wiseman and G. J. Milburn, Quantum Measurement and Control (Cambridge University Press, Cambridge, 2009). 